\title{
ROLE OF PHYCOREMEDIATION FOR NUTRIENT REMOVAL FROM WASTEWATERS: A REVIEW
}

\author{
EMPARAN, Q. ${ }^{1}-$ HARUN, R. ${ }^{*}-$ DANQUAH, M. K. ${ }^{2}$ \\ ${ }^{1}$ Department of Chemical and Environmental Engineering, Faculty of Engineering \\ Universiti Putra Malaysia, 43400 Serdang, Malaysia \\ ${ }^{2}$ Department of Chemical and Petroleum Engineering, Curtin University \\ 98009 Miri, Sarawak, Malaysia \\ *Corresponding author \\ e-mail:mh_razif@upm.edu.my; phone: +60389466289; fax: +60386567120
}

(Received 26 ${ }^{\text {th }}$ Sep 2018; accepted 22 ${ }^{\text {nd }}$ Nov 2018)

\begin{abstract}
The presence of high concentrations of chemical oxygen demand (COD), biochemical oxygen demand (BOD) and nutrients in wastewater generated industrially or domestically has resulted in significant water pollution situations and subsequently is leading to adverse health problems. Algae have been used in various applications in environmental biotechnology especially for phycoremediation as a tertiary wastewater treatment strategy through assimilation of high concentration of nitrogen and phosphorus for their growth, thus reducing potential eutrophication problems. This article discusses the role of phycoremediation to remove COD, BOD and nutrients from wastewater. The mechanism for nutrient removal from wastewater, challenges to process development and current commercial-scale algae-based wastewater treatment are reviewed too. It appears that phycoremediation plays a vital role to treat wastewaters efficiently.
\end{abstract}

Keywords: microalgae, wastewater pollution, COD, BOD, efficiency

\section{Introduction}

In the last few decades, the rapid population growth, industrial revolution, and urbanization have led to various forms of environmental pollution. The disposal of untreated wastewater (e.g. industrial, municipal, palm oil mill effluent, amongst others) directly into water bodies such as rivers, lakes, and oceans is considered a simple and cheap discharge method in communities where wastewater disposal is not well regulated (Chan et al., 2009). This contributes significantly to water shortage. In addition, the potential of high concentrations of toxic pollutants moves into human and animal food chain could result in significant health problems (Barakat, 2011).

The quality of water will degrade when untreated wastewater discharged into the receiving water body (e.g. rivers, streams, lakes) and lead to the problem of clean water for human consumption. Besides that, the discharged of wastewater containing the excessive amount of nutrients (e.g. nitrogen, phosphorus) into the receiving water body can also lead to another problem like eutrophication resulted in the depletion of oxygen level in the water (Lau et al., 1997). Generally, phosphorus in the form of orthophosphates is known as the limiting nutrient in the freshwater system. However, runoff wastewater containing extremely concentration of phosphorus can lead to the eutrophication (Cai et al., 2013). The abnormally low level of oxygen in the water body can harm aquatic life by inducing the reduction of aquatic animals (e.g. fishes, prawns, among others) and microorganisms (e.g. bacteria, fungi, algae) population (Sperling and Chernicharo, 2005). 
According to Driscoll et al. (2003) and Smith (2003), the eutrophication in fresh and coastal or marine ecosystems also leads to some problems such as water discoloration and foaming and increasing in blooms of toxic algal species and their biomass, mortality rate of aquatic species, sedimentation of organic particles as well as decreasing in water transparency.

Nitrate occurs naturally in water; however, it is undesirable substance in public water because of its high concentration in drinking water may either cause serious health problems like methemoglobinemia (blue-baby syndrome) or source of nitrosamines after its reduction to nitrite (Schoeman and Steyn, 2003; Abdel-Raouf et al., 2012). According to Abdel-Raouf et al. (2012) also stated that the purpose of removing phosphate from wastewater is to protect water from eutrophication.

Treated wastewaters obtained from conventional treatments remain undesirable for discharge because of their characteristics still not able to meet the standards set by local authorities (Loh et al., 2013). Therefore, more effective treatment technologies are required in order to reduce the exposure of toxic chemicals to natural ecosystems. The use of microalgae to treat wastewater and hazardous contaminants is currently of global interest due to the effective photosynthetic uptake of high concentrations of minerals, ionogens, and organics by microalgae, and the capacity to simultaneously utilize carbon dioxide $\left(\mathrm{CO}_{2}\right)$ (Mohan et al., 2001; Zeng et al., 2012). Microalgae cells have the ability to remove nutrients such as phosphorus, nitrogen, ammonium as well as heavy metals in wastewater (Phang and Ong, 1988; Aziz and Ng, 1992; Sydney et al., 2011; AbdelRaouf et al., 2012). A life cycle economic assessment has shown that, for microalgae cultivation, the growth media formulation and composition contribute significantly to the operating cost and is a major consideration for scale-up design (Clarens et al., 2009; Lam and Lee, 2011). Hence there is the need to look into cheaper nutrients sources, and wastewaters containing the right nutrients compositions could be a viable alternative. This will also reduce the cost of microalgae biomass generation for the production of biofuels, animal feed, and essential oils amongst others. Meanwhile, the microalgae biomass production after treatment of heavy metal from wastewaters can be used as a potential feedstock for biochar, charcoal, biofuel and biogas production (Safonova et al., 2004; Chinnasamy et al., 2010; Poo et al., 2018). Therefore, the aims of this paper are to comprehensively review the current use of both free cells and immobilized algae in treating wastewaters to obtain some new ideas to deal with wastewaters without having a negative effect on the environment.

\section{Microalgae and macroalgae}

Algae are aquatic plant-like organisms (phytoplankton) with various shapes. They lack roots, stems, and leaves, with cell walls made of cellulose. Algae cells are divided into macroalgae and microalgae. Macroalgae are multicellular organisms with size up to several meters while microalgae are small organisms (unicellular) with their size in the range 0.2-100 $\mu \mathrm{m}$ (Bhatt et al., 2014).

Sharma et al. (2011) categorized microalgae into several groups: (i) prokaryotic bluegreen (cyanobacteria); (ii) eukaryotic green (Chlorophyceae); (iii) eukaryotic brown (Phaeophyceae); (iv) eukaryotic red (Rhodophyceae); and (v) eukaryotic diatoms (Bacillariophceae) as shown in Table 1.

According to Brennan and Owende (2010) and Mata et al. (2010), algae are photosynthetic prokaryotic or eukaryotic microorganisms that can grow rapidly and 
have the ability to adapt to harsh environments due to their unicellular or simple multicellular structure. They are thallophytes containing chlorophyll as their main photosynthetic pigment. In fact, microalgae have more effective access to carbon dioxide, water $\left(\mathrm{H}_{2} \mathrm{O}\right)$ and nutrients due to their simple cellular structure compared to terrestrial plants. As a result, they are known to have very high carbon capturing and photosynthetic efficiencies with the ability to convert solar energy into useful biomass and reduce $\mathrm{CO}_{2}$ concentrations in the atmosphere more efficiently than terrestrial plants (Packer, 2009; Kumar et al., 2013).

Table 1. Classification of microalgae and related species (Packer, 2009)

\begin{tabular}{c|c}
\hline Algae group & Microalgae species \\
\hline $\begin{array}{c}\text { Prokaryotic blue-green } \\
\text { (Cyanobacteria })\end{array}$ & Arthrospira, Gloeocapsa, Microcystis, Oscillatoria, etc. \\
\hline $\begin{array}{c}\text { Eukaryotic green } \\
\text { (Chlorophyceae })\end{array}$ & Botryococcus, Chlamydomonas, Chlorella, Scenedesmus, etc. \\
\hline $\begin{array}{c}\text { Eukaryotic brown } \\
(\text { Phaeophyceae })\end{array}$ & Dinobryon, Mallamonas, Ochromonas, Synura, Uroglena etc. \\
\hline $\begin{array}{c}\text { Eukaryotic red } \\
(\text { Rhodophyceae })\end{array}$ & Porphyridium \\
\hline $\begin{array}{c}\text { Eukaryotic diatoms } \\
(\text { Bacillariophyceae })\end{array}$ & Asterionella, Cyclotella, Fragilaria, Surirella, etc. \\
\hline
\end{tabular}

\section{Metabolism of microalgae}

Generally, the growth of microalgae biomass depends on carbon source and photons to perform photosynthesis (Costa and de Morais, 2013). Microalgae can modify their internal structure by both biochemical and physiological acclimations. Externally, they can also excrete various compounds to other cells, supply nutrients or limit the growth of competitors.

Autotrophic microalgae use inorganic compounds and sunlight as a carbon and energy source. In the presence of light, these autotrophic microalgae are referred to as photoautotrophic since they use light photons as an energy source to generate chemical energy by photosynthesis (Amaro et al., 2011). During photosynthesis by autotrophic algae, $\mathrm{CO}_{2}$ and water are converted into carbohydrate (glucose) and further metabolized to yield energy which drives the formation of adenosine triphosphate (ATP) from adenosine diphosphate (ADP). The energy in ATP is then used to drive various processes in the cells, and in doing so is converted back to ADP ready to pick up more energy to enable growth (Brennan and Owende, 2010). Heterotrophic microalgae use solely organic compounds and exogenous nutrients as a source of carbon and energy for growth in dark conditions (Amaro et al., 2011). According to Huang et al. (2010), cultivation of heterotrophic microalgae overcomes problems associated with limited light photons that affect the attainment of high cells densities during photosynthesis. Some microalgae are mixotrophic, with the capacity to exist as autotrophic or heterotrophic depending on the concentration of organic compound and also the availability of light (Chojnacka and Noworyta, 2004). 


\section{Phycoremediation}

Microalgae have been used in various applications of environmental biotechnology especially for bioremediation (e.g. phycoremediation). Bioremediation is the part of environmental biotechnology that uses a biological process to treat contaminants (Boopathy, 2000). Gani et al. (2015a), Rao et al. (2011), and Olguin (2003) defined phycoremediation as the use of algae to remove or transform pollutants, including nutrients and toxic chemicals from wastewater and $\mathrm{CO}_{2}$ from waste air together with biomass production. Wastewaters treatment by microalgae can be performed in the form of suspended free-cells culture and immobilized cells. The suspended free-cells culture is the condition of microalgae living cells move independently within the bottles containing medium under a condition to ensure uniform cells distribution (Katarzyna et al., 2015). Meanwhile, the immobilized cells is the condition of microalgae living cells be prevented from flow freely from its original location to all parts of the medium. This approach can be performed by keeping the microalgae living cells in the carriers such as NaCS-PDMDAAC capsules (Zeng et al., 2012), alginate (Sumithrabhai et al., 2016) and chitosan beads (Fierro et al., 2008).

The use of suspended free-microalgae cells culture to treat wastewater was first studied by Oswald et al. (1957). The process involves the removal of nitrogen and phosphorus from wastewater whilst simultaneously providing oxygen $\left(\mathrm{O}_{2}\right)$ for aerobic bacteria coexisting in the culture. Biologically treating wastewater using microalgae is a reliable process due to the high photosynthetic efficiency and growth rates. Furthermore, microalgae have effective nutrient uptake capacity with the potential to achieve great removal of nitrogen and phosphorus as well as heavy metals from wastewater (Hernandez et al., 2006; Hameed, 2007; Sengar et al., 2011; Abdel-Raouf et al., 2012).

Microalgae require significant amounts of phosphorus and nitrogen for proteins synthesis (45-60\% microalgae dry weight), nucleic acids and phospholipids for their growth (Rao et al., 2011). In this respect, nutrient removal using microalgae presents major prospects for tertiary wastewater treatment aimed at removing ammonia, nitrate, and phosphate (Rawat et al., 2011; Abdel-Raouf et al., 2012; Gani et al., 2015b). After nutrient uptake by microalgae during wastewater treatment, the purified water can be decanted to harvest the free cells microalgae (Abdel-Raouf et al., 2012).

Phycoremediation has been used in various applications: (i) removal of nutrients from organic matter-rich wastewater; (ii) removal of nutrients and xenobiotic compounds using algae-derived sorbents; (iii) treatment of heavy metal-rich wastewater; (iv) sequestration of $\mathrm{CO}_{2}$; (v) transformation and degradation of xenobiotic; and (vi) detection of toxic compounds using algae-based biosensors. Application of phycoremediation for wastewater treatment has significant benefits (Eroglu et al., 2012; Sivakumar and Rajendran, 2013; Whangchenchom et al., 2014; Gani et al., 2015a). The following are key characteristics of the process.

i. It is cost-effective, eco-friendly and safe.

ii. Microalgae used are non-pathogenic photosynthetic organisms and produce nontoxic substances.

iii. It efficiently reduces nutrient load and leads to a reduction in total dissolved solid.

iv. It detoxifies and removes pollutants (e.g. heavy metals) from toxic waste-rich sludge more effectively than conventional chemical treatment technologies. 
v. It increases dissolved oxygen (DO) levels via photosynthetic activity.

vi. Microalgae use $\mathrm{CO}_{2}$ fixation from the atmosphere as a source of carbon for growth thus reduce greenhouse gasses (GHG).

vii. Production of high-value products derived from nutrient-rich microalgae biomass for bio-fertilizer production and as feed for animals and aquaculture.

viii. Simple operation and maintenance.

ix. Construction and operation costs are cheaper than mechanical treatment plants such as activated sludge and sequencing batch reactors.

$\mathrm{x}$. Sustainable treatment solution with significant potential for energy and nutrient recovery.

Figure 1 shows the schematics of mixed microbial community-based treatment of wastewater exploiting the metabolic relationship between microalgae and bacteria. First, the bacteria proliferate and produce $\mathrm{CO}_{2}$ for microalgae growth. The $\mathrm{CO}_{2}$ is used by the microalgae during photosynthesis in the presence of light to produce $\mathrm{O}_{2}$ which is assimilated by the bacteria for growth. According to Sharma and Khan (2013), microalgae produce oxygen from water as a by-product of photosynthesis and bacteria use the oxygen to oxidize organic compounds. During photosynthesis, the end product of bio-oxidation of organic compounds namely, carbon dioxide, is further fixed into cells carbon by microalgae. As a result, the pollutants level in wastewater are reduced to undetectable or acceptable limits set by local authorities.

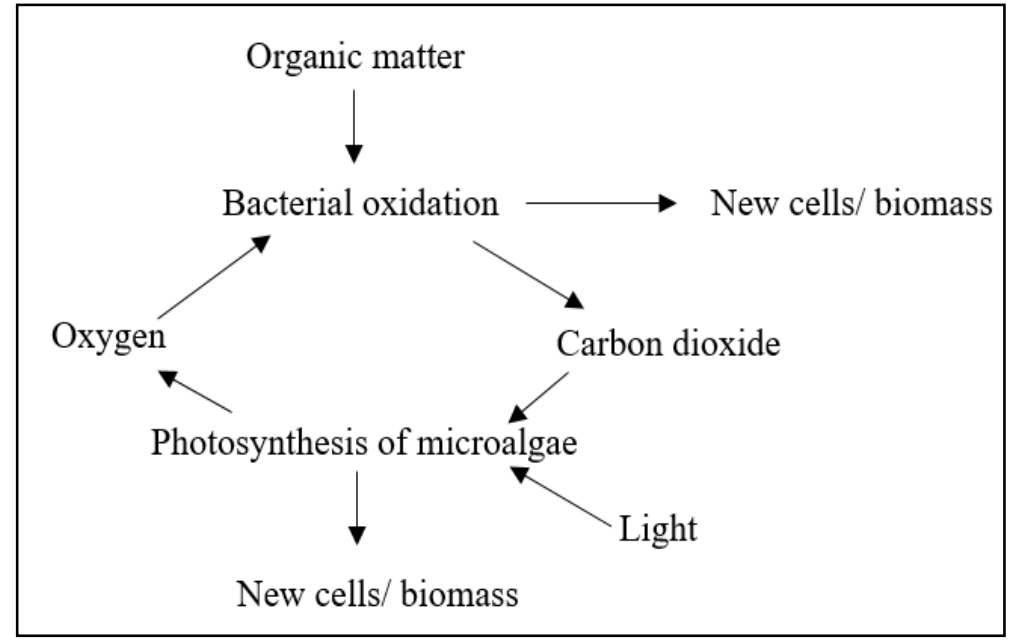

Figure 1. BOD removal through the photosynthetic oxygenation approach (Gani et al., 2015a)

Generally, the generated microalgae biomass from wastewater treatment process is used for agriculture (fertilizer and soil conditioners) and biofuels industries (Eroglu et al., 2012). In this respect, the cultivation of microalgae using wastewater serves a dual role of pollutants load reduction and production of various valuable products.

\section{Mechanisms of carbon, nitrogen and phosphorus removal in phycoremediation}

Elements like carbon, nitrogen, phosphorus and, sulfur together with small amounts of trace metals (e.g. sodium, calcium, iron etc.) are required for algae growth. Amongst 
these elements, uptake of nitrogen and phosphorus is critical for algal growth (Cai et al., 2013).

Autotrophic microalgae fix carbon (in the form of $\mathrm{CO}_{2}$ ) biologically from the atmosphere by photosynthesis. Microalgae can also use carbon in the form of soluble carbonates for their growth, either by direct uptake or conversion of carbonate to free $\mathrm{CO}_{2}$ through a carboanhydrase activity (Cai et al., 2013).

Nitrogen in wastewater is present in the form of $\mathrm{NH}_{4}{ }^{+}$(ammonia), $\mathrm{NO}_{2}{ }^{-}$(nitrite) and $\mathrm{NO}_{3}{ }^{-}$(nitrate) (Hadiyanto et al., 2013). The conversion of inorganic nitrogen into organic forms can be carried out by eukaryotic microalgae via assimilation (Cai et al., 2013). Figure 2 shows the steps involved in the conversion of inorganic nitrogen into organic forms. Firstly, translocation of inorganic nitrogen takes places across the plasma membrane of the algae cells with subsequent reduction to nitrate and nitrite by nitrate and nitrite reductase, respectively. The next step is the conversion of ammonium into amino acids (glutamine). Nitrate reductase utilizes the reduced form of nicotinamide adenine dinucleotide (NADH) to transfer two electrons in the reaction for the conversion of nitrate into nitrite. Next, nitrite is further reduced to ammonium by nitrite reductase and ferredoxin $(\mathrm{Fd})$ to transfer six electrons in the reaction. All inorganic forms of nitrogen are reduced to ammonium before being incorporated into amino acids within the intracellular fluid. Finally, glutamine synthase using glutamate (Glu) and adenosine triphosphate (ATP) facilitates the incorporation ammonium into amino acids (glutamine) (Cai et al., 2013).

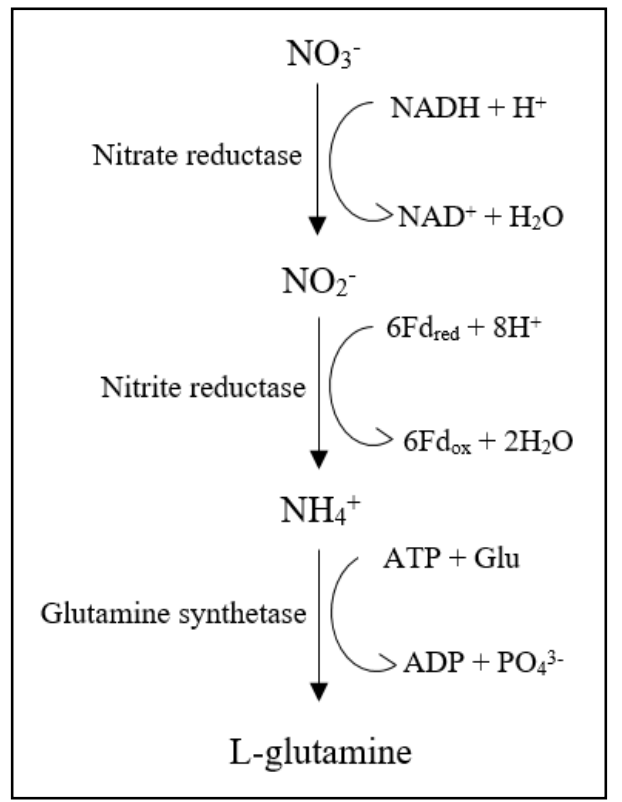

Figure 2. Schematic of the conversion of inorganic nitrogen to its organic form via assimilation (Cai et al., 2013)

The phosphorus present in lipids, nucleic acids, and proteins as well as intermediates of carbohydrate metabolism is a result of phosphorus uptake. Inorganic phosphorus in the form of phosphates plays a crucial role in the growth of algae cells and also their energy metabolism. According to Martínez et al. (1999), algae metabolism relies mostly on inorganic phosphorus in the forms of hydrogen phosphate $\left(\mathrm{HPO}_{4}{ }^{2-}\right)$ and dihydrogen 
phosphate $\left(\mathrm{H}_{2} \mathrm{PO}_{4}\right)$ which is then incorporated into organic compounds through a phosphorylation process involving the production of ADP-derived ATP together with energy input. The oxidation of respiratory substrates, electron transport system of mitochondria, or light (photosynthesis) is all sources of energy input. Phosphates are transferred across the plasma membrane of the algal cells for utilization.

The nitrate and phosphate in the wastewater are adsorbed through the matrix pore surface of microcapsules membrane. After that, the uptake and assimilation of nitrate and phosphate ion by immobilized microalgae cells for growth occur and result in the reduction of $\mathrm{N}$ and $\mathrm{P}$ content in wastewater and further improves the quality of wastewater for discharge (Zeng et al., 2012).

\section{Selection of microalgae species for phycoremediation}

The selection of microalgae species for wastewater treatment is a critical consideration. According to Shi et al. (2007) and Olguin (2003), the selection of microalgae species for wastewater treatment should consider its robustness against wastewater pollutants, the capability to grow well (high growth rates), and their efficiency in assimilating nutrient from wastewater. A lot of studies focusing on various species of microalgae cultivated in wastewater for the removal of nitrogen and phosphorus have been reported. Scenedesmus, Chlorella, and Botryococcus are commonly used microalgae for removing nutrients (nitrogen and phosphorus), COD and BOD as shown in Table 2.

Table 2. Comparison of phycoremediation efficiency by different microalgae species (suspended free-cells microalgae cultures) grown in the various wastewater effluent

\begin{tabular}{|c|c|c|c|c|}
\hline $\begin{array}{l}\text { Microalgae } \\
\text { species }\end{array}$ & Source of the wastewater (ww) & Parameter & $\begin{array}{c}\text { Removal } \\
\text { efficiency } \\
(\%)\end{array}$ & Ref. \\
\hline \multirow{8}{*}{ Chlorella sp. } & \multirow{4}{*}{ Synthetic aquaculture wastewater } & $\mathrm{COD}$ & $\mathrm{COD}=15 \%$ & \multirow{4}{*}{$\begin{array}{l}\text { Udom et al., } \\
\text { (2013) }\end{array}$} \\
\hline & & $\mathrm{TN}$ & $\mathrm{TN}=91 \%$ & \\
\hline & & $\mathrm{NH}_{4}^{+}$ & $\mathrm{NH}_{4}^{+}=100 \%$ & \\
\hline & & $\mathrm{TP}$ & $\mathrm{TP}=93 \%$ & \\
\hline & \multirow{4}{*}{$\begin{array}{l}\text { Domestic wastewater before the primary } \\
\text { settling }\end{array}$} & COD & $\begin{array}{l}\mathrm{COD}= \\
50.90 \%\end{array}$ & \multirow{4}{*}{$\begin{array}{l}\text { Wang et al., } \\
\text { (2010) }\end{array}$} \\
\hline & & $\mathrm{TN}$ & $\mathrm{TN}=68.40 \%$ & \\
\hline & & $\mathrm{NH}_{4}^{+}$ & $\begin{array}{l}\mathrm{NH}_{4}^{+}= \\
82.40 \%\end{array}$ & \\
\hline & & $\mathrm{PO}_{4}{ }^{3-}$ & $\begin{array}{l}\mathrm{PO}_{4}{ }^{3-}= \\
83.20 \%\end{array}$ & \\
\hline \multirow{11}{*}{$\begin{array}{l}\text { Chlorella } \\
\text { vulgaris }\end{array}$} & \multirow{4}{*}{$\begin{array}{l}\text { Sewage wastewater collected from the } \\
\text { treatment plant }\end{array}$} & BOD & $\mathrm{BOD}=70 \%$ & \multirow{4}{*}{$\begin{array}{l}\text { Abou-Shanab et } \\
\text { al., (2013) }\end{array}$} \\
\hline & & COD & $\mathrm{COD}=66 \%$ & \\
\hline & & TN & $\mathrm{TN}=71 \%$ & \\
\hline & & TP & $\mathrm{TP}=67 \%$ & \\
\hline & \multirow{2}{*}{ Treated piggery wastewater } & $\mathrm{TN}$ & $\mathrm{TN}=49 \%$ & \multirow{2}{*}{$\begin{array}{l}\text { Abou-Shanab et } \\
\text { al., (2013) }\end{array}$} \\
\hline & & $\mathrm{TP}$ & $\mathrm{TP}=18 \%$ & \\
\hline & \multirow{5}{*}{$\begin{array}{c}\text { Sewage wastewater collected from the } \\
\text { various drains }\end{array}$} & BOD & $\begin{array}{l}\mathrm{BOD}= \\
98.70 \%\end{array}$ & \multirow{5}{*}{$\begin{array}{l}\text { Ahmad et al., } \\
\text { (2013) }\end{array}$} \\
\hline & & COD & $\begin{array}{l}\text { COD = } \\
98.30 \%\end{array}$ & \\
\hline & & TKN & $\begin{array}{l}\mathrm{TKN}= \\
93.10 \%\end{array}$ & \\
\hline & & $\mathrm{NO}_{3}^{-}$ & $\begin{array}{l}\mathrm{NO}_{3}^{-}= \\
98.30 \%\end{array}$ & \\
\hline & & $\mathrm{TP}$ & $\mathrm{TP}=98 \%$ & \\
\hline
\end{tabular}




\begin{tabular}{|c|c|c|c|c|}
\hline $\begin{array}{l}\text { Microalgae } \\
\text { species }\end{array}$ & Source of the wastewater (ww) & Parameter & $\begin{array}{c}\text { Removal } \\
\text { efficiency } \\
(\%)\end{array}$ & Ref. \\
\hline & & $\mathrm{PO}_{4}^{+}$ & $\begin{array}{l}\mathrm{PO}_{4}^{+}= \\
98.60 \% \\
\end{array}$ & \\
\hline & \multirow{2}{*}{$\begin{array}{l}\text { Drainage solution from the commercial } \\
\text { green production }\end{array}$} & TN & $\mathrm{TN}=20.70 \%$ & \multirow{2}{*}{$\begin{array}{l}\text { Hultberg et al., } \\
\text { (2013) }\end{array}$} \\
\hline & & $\mathrm{TP}$ & $\mathrm{TP}=99.70 \%$ & \\
\hline & \multirow{4}{*}{$\begin{array}{l}\text { Sewage wastewater collected from the } \\
\text { treatment plant }\end{array}$} & BOD & $\begin{array}{l}\mathrm{BOD}= \\
70.91 \%\end{array}$ & \multirow{4}{*}{$\begin{array}{l}\text { Kshirsagar, } \\
\text { (2013) }\end{array}$} \\
\hline & & COD & $\begin{array}{l}\text { COD = } \\
80.64 \%\end{array}$ & \\
\hline & & $\mathrm{NO}_{3}$ & $\begin{array}{l}\mathrm{NO}_{3}= \\
78.08 \%\end{array}$ & \\
\hline & & $\mathrm{PO}_{4}^{+}$ & $\begin{array}{l}\mathrm{PO}_{4}^{+}= \\
79.66 \%\end{array}$ & \\
\hline & \multirow{4}{*}{$\begin{array}{l}\text { Sewage wastewater collected from the } \\
2^{\text {nd }} \text { clarifier treatment plant }\end{array}$} & $\mathrm{NO}_{3}{ }^{-}$ & $\mathrm{NO}_{3}^{-}=99 \%$ & \multirow{4}{*}{ Su et al., (2012) } \\
\hline & & $\mathrm{NO}_{2}^{-}$ & $\mathrm{NO}_{2}{ }^{-}=99 \%$ & \\
\hline & & $\mathrm{NH}_{4}^{+}$ & $\mathrm{NH}_{4}^{+}=99 \%$ & \\
\hline & & $\mathrm{PO}_{4}^{3-}$ & $\mathrm{PO}_{4}{ }^{3-}=99 \%$ & \\
\hline & \multirow{2}{*}{$\begin{array}{l}\text { Sewage wastewater collected from the } \\
\text { primary settling tank }\end{array}$} & $\mathrm{NH}_{4}^{+}$ & $\mathrm{NH}_{4}{ }^{+}=50 \%$ & \multirow{2}{*}{$\begin{array}{l}\text { Lau et al., } \\
\text { (1997) }\end{array}$} \\
\hline & & $\mathrm{PO}_{4}{ }^{3-}$ & $\mathrm{PO}_{4}^{3-}=50 \%$ & \\
\hline & Synthetic sewage & $\mathrm{NO}_{3}^{-}$ & $\mathrm{NO}_{3}^{-}=87 \%$ & $\begin{array}{l}\text { Eroglu et al., } \\
(2012)\end{array}$ \\
\hline & \multirow{7}{*}{$\begin{array}{l}\text { Leather processing collected from the } \\
\text { manufacturing facility }\end{array}$} & BOD & $\mathrm{BOD}=22 \%$ & \multirow{7}{*}{$\begin{array}{l}\text { Rao et al., } \\
\text { (2011) }\end{array}$} \\
\hline & & COD & $\mathrm{COD}=38 \%$ & \\
\hline & & TKN & $\mathrm{TKN}=73 \%$ & \\
\hline & & $\mathrm{NO}_{3}^{-}$ & $\begin{array}{l}\mathrm{NO}_{3}{ }^{-}= \\
91.49 \%\end{array}$ & \\
\hline & & $\mathrm{NO}_{2}^{-}$ & $\mathrm{NO}_{2}^{-}=89 \%$ & \\
\hline & & $\mathrm{NH}_{4}^{+}$ & $\mathrm{NH}_{4}^{+}=80 \%$ & \\
\hline & & $\mathrm{PO}_{4}$ & $\mathrm{PO}_{4}=94 \%$ & \\
\hline & \multirow{3}{*}{$\begin{array}{l}\text { Textile wastewater (garment factory) } \\
\text { collected from the holding tank }\end{array}$} & COD & $\begin{array}{l}\mathrm{COD}= \\
62.30 \% \\
\end{array}$ & \multirow{3}{*}{$\begin{array}{l}\text { Lim et al., } \\
\quad(2010)\end{array}$} \\
\hline & & $\mathrm{NH}_{4}^{+}$ & $\begin{array}{l}\mathrm{NH}_{4}^{+}= \\
45.10 \%\end{array}$ & \\
\hline & & $\mathrm{PO}_{4}^{+}$ & $\begin{array}{l}\mathrm{PO}_{4}^{+}= \\
33.30 \% \\
\end{array}$ & \\
\hline & \multirow{3}{*}{$\begin{array}{l}\text { Chemical (based products) wastewater } \\
\text { collected from the Periyor }\end{array}$} & $\mathrm{NO}_{3}^{-}$ & $\mathrm{NO}_{3}{ }^{-}=84 \%$ & \multirow{3}{*}{$\begin{array}{l}\text { Dominic et al., } \\
\text { (2009) }\end{array}$} \\
\hline & & $\mathrm{NO}_{2}^{-}$ & $\mathrm{NO}_{2}^{-}=100 \%$ & \\
\hline & & $\mathrm{PO}_{4}^{3-}$ & $\begin{array}{l}\mathrm{PO}_{4}{ }^{3-}= \\
69.23 \%\end{array}$ & \\
\hline & \multirow{2}{*}{ Sewage wastewater } & COD & $\mathrm{COD}=78 \%$ & \multirow{2}{*}{$\begin{array}{c}\text { Kumar et al., } \\
\text { (2018) }\end{array}$} \\
\hline & & $\mathrm{NO}_{3}^{-}$ & $\mathrm{NO}_{3}^{-}=75 \%$ & \\
\hline \multirow{3}{*}{ Chlorella sakina } & \multirow{3}{*}{ Tannery wastewater } & $\mathrm{NO}_{3}^{-}$ & $\mathrm{NO}_{3}{ }^{-}=9.11 \%$ & \multirow{3}{*}{$\begin{array}{c}\text { Jaysudha and } \\
\text { Sampathkumar, } \\
\text { (2014) }\end{array}$} \\
\hline & & $\mathrm{NH}_{4}^{+}$ & $\begin{array}{l}\mathrm{NH}_{4}^{+}= \\
62.04 \%\end{array}$ & \\
\hline & & $\mathrm{PO}_{4}^{3-}$ & $\begin{array}{l}\mathrm{PO}_{4}^{3-}= \\
81.94 \%\end{array}$ & \\
\hline \multirow{4}{*}{$\begin{array}{c}\text { Chlorella } \\
\text { pyrenoidosa }\end{array}$} & \multirow{4}{*}{$\begin{array}{l}\text { Dairy wastewater collected from the } \\
\text { farm }\end{array}$} & BOD & $\mathrm{BOD}=88 \%$ & \multirow{4}{*}{$\begin{array}{l}\text { Yadavalli et al., } \\
\text { (2013) }\end{array}$} \\
\hline & & COD & $\mathrm{COD}=85 \%)$ & \\
\hline & & $\mathrm{NH}_{4}^{+}$ & $\mathrm{NH}_{4}^{+}=98 \%$ & \\
\hline & & $\mathrm{PO}_{4}^{3-}$ & $\mathrm{PO}_{4}^{3-}=98 \%$ & \\
\hline \multirow{3}{*}{$\begin{array}{l}\text { Chlorella } \\
\text { zofingiensis }\end{array}$} & \multirow{3}{*}{$\begin{array}{l}\text { Piggery wastewater collected from the } \\
\text { private farm }\end{array}$} & COD & $\begin{array}{l}\mathrm{COD}= \\
79.84 \%\end{array}$ & \multirow{3}{*}{$\begin{array}{l}\text { Zhu et al., } \\
\text { (2013) }\end{array}$} \\
\hline & & $\mathrm{TN}$ & $\mathrm{TN}=82.70 \%$ & \\
\hline & & $\mathrm{TP}$ & $\mathrm{TP}=98.17 \%$ & \\
\hline
\end{tabular}




\begin{tabular}{|c|c|c|c|c|}
\hline $\begin{array}{l}\text { Microalgae } \\
\text { species }\end{array}$ & Source of the wastewater (ww) & Parameter & $\begin{array}{c}\text { Removal } \\
\text { efficiency } \\
(\%)\end{array}$ & Ref. \\
\hline \multirow{5}{*}{$\begin{array}{l}\text { Chlorella } \\
\text { minutissima }\end{array}$} & \multirow{5}{*}{ Primary treated domestic wastewater } & BOD & $\mathrm{BOD}=95 \%$ & \multirow{5}{*}{$\begin{array}{l}\text { Sharma and } \\
\text { Khan, (2013) }\end{array}$} \\
\hline & & COD & $\mathrm{COD}=90 \%$ & \\
\hline & & $\mathrm{NO}_{3}^{-}$ & $\begin{array}{l}\mathrm{NO}_{3}^{-}= \\
91.49 \%\end{array}$ & \\
\hline & & $\mathrm{NH}_{4}^{+}$ & $\mathrm{NH}_{4}^{+}=90 \%$ & \\
\hline & & $\mathrm{PO}_{4}^{3-}$ & $\begin{array}{l}\mathrm{PO}_{4}^{3-}= \\
74.27 \%\end{array}$ & \\
\hline \multirow[b]{2}{*}{$\begin{array}{l}\text { Chlorella } \\
\text { sorokoniana }\end{array}$} & Synthetic municipal wastewater & $\mathrm{PO}_{4}{ }^{3-}$ & $\mathrm{PO}_{4}{ }^{3-}=69 \%$ & \multirow[b]{2}{*}{$\begin{array}{l}\text { Hernandez et } \\
\text { al., (2006) }\end{array}$} \\
\hline & $\begin{array}{l}\text { Municipal wastewater collected from the } \\
\text { aerobic activated sludge }\end{array}$ & $\mathrm{PO}_{4}^{3-}$ & $\mathrm{PO}_{4}{ }^{3-}=72 \%$ & \\
\hline \multirow{2}{*}{ Chlorella sp. } & \multirow{2}{*}{$\begin{array}{l}\text { Mixed wastewaters from piggery and } \\
\text { winery }\end{array}$} & $\mathrm{TN}$ & $\mathrm{TN}=89 \%$ & \multirow{2}{*}{$\begin{array}{l}\text { Ganeshkumar } \\
\text { et al., (2018) }\end{array}$} \\
\hline & & $\mathrm{TP}$ & $\mathrm{TP}=49 \%$ & \\
\hline \multirow{4}{*}{$\begin{array}{l}\text { Chlamydomonas } \\
\text { sp. }\end{array}$} & \multirow{4}{*}{$\begin{array}{l}\text { POME collected from the facultative } \\
\text { pond }\end{array}$} & COD & $\begin{array}{l}\mathrm{COD}= \\
29.13 \%\end{array}$ & \multirow{4}{*}{$\begin{array}{l}\text { Ding et al., } \\
\text { (2016) }\end{array}$} \\
\hline & & $\mathrm{TN}$ & $\mathrm{TN}=72.97 \%$ & \\
\hline & & $\mathrm{NH}_{4}^{+}$ & $\mathrm{NH}^{+}=100 \%$ & \\
\hline & & TP & $\mathrm{TP}=63.53 \%$ & \\
\hline \multirow{5}{*}{ Scenedesmus sp. } & $\begin{array}{c}\text { Noodle processing - MLSS (aeration } \\
\text { tank) }\end{array}$ & COD & $\begin{array}{l}\mathrm{COD}= \\
71.85 \%\end{array}$ & \multirow{2}{*}{$\begin{array}{l}\text { Whangchencho } \\
\text { m et al., (2014) }\end{array}$} \\
\hline & $\begin{array}{c}\text { Noodle processing - effluent (final } \\
\text { sedimentation tanks) }\end{array}$ & COD & $\begin{array}{l}\mathrm{COD}= \\
39.89 \%\end{array}$ & \\
\hline & Primary treated domestic wastewater & $\mathrm{NH}_{4}$ & $\mathrm{NH}_{4}=90 \%$ & $\begin{array}{l}\text { Sharma and } \\
\text { Khan, (2013) }\end{array}$ \\
\hline & \multirow{2}{*}{$\begin{array}{l}\text { Synthetic } 2 \mathrm{f} \text { medium with } 44 \mathrm{mg} / \mathrm{L} \\
\text { nitrate and } 6 \mathrm{mg} / \mathrm{L} \text { phosphate }\end{array}$} & $\mathrm{NO}_{3}^{-}$ & $\mathrm{NO}_{3}{ }^{-}=20 \%$ & \multirow{2}{*}{$\begin{array}{l}\text { Fierro et al., } \\
(2008)\end{array}$} \\
\hline & & $\mathrm{PO}_{4}{ }^{3-}$ & $\mathrm{PO}_{4}{ }^{3-}=30 \%$ & \\
\hline \multirow{8}{*}{$\begin{array}{l}\text { Scenedesmus } \\
\text { obliquus }\end{array}$} & \multirow{6}{*}{ Treated piggery wastewater } & TN & $\mathrm{TN}=58 \%$ & \multirow{2}{*}{$\begin{array}{l}\text { Abou-Shanab et } \\
\text { al., (2013) }\end{array}$} \\
\hline & & $\mathrm{TP}$ & $\mathrm{TP}=24 \%$ & \\
\hline & & $\mathrm{TN}$ & $\mathrm{TN}=60 \%$ & \multirow{4}{*}{$\begin{array}{l}\text { Jimenez-Perez } \\
\text { et al., (2004) }\end{array}$} \\
\hline & & $\mathrm{NO}_{3}^{-}$ & $\mathrm{NO}_{3}{ }^{-}=84 \%$ & \\
\hline & & $\mathrm{NH}_{4}^{+}$ & $\mathrm{NH}_{4}^{+}=57 \%$ & \\
\hline & & $\mathrm{TP}$ & $\mathrm{TP}=83 \%$ & \\
\hline & \multirow[t]{2}{*}{ Synthetic brewery effluent } & COD & $\begin{array}{l}\mathrm{COD}= \\
57.50 \%\end{array}$ & \multirow{2}{*}{$\begin{array}{l}\text { Mata et al., } \\
\text { (2012) }\end{array}$} \\
\hline & & $\mathrm{TN}$ & $\mathrm{TN}=20.80 \%$ & \\
\hline \multirow{4}{*}{$\begin{array}{l}\text { Scenedesmus } \\
\text { acutus }\end{array}$} & \multirow{4}{*}{$\begin{array}{c}\text { Municipal wastewater effluent was } \\
\text { collected from the conventional activated } \\
\text { sludge plant }\end{array}$} & COD & $\begin{array}{l}\mathrm{COD}= \\
77.30 \%\end{array}$ & \multirow{4}{*}{$\begin{array}{l}\text { Sacristán de } \\
\text { Alva et al., } \\
\text { (2013) }\end{array}$} \\
\hline & & $\mathrm{NO}_{3}^{-}$ & $\begin{array}{l}\mathrm{NO}_{3}^{-}= \\
71.10 \%\end{array}$ & \\
\hline & & $\mathrm{NH}_{4}^{+}$ & $\begin{array}{l}\mathrm{NH}_{4}^{+}= \\
93.60 \%\end{array}$ & \\
\hline & & $\mathrm{PO}_{4}^{3-}$ & $\begin{array}{l}\mathrm{PO}_{4}^{3-}= \\
66.20 \%\end{array}$ & \\
\hline \multirow{4}{*}{$\begin{array}{l}\text { Scenedesmus } \\
\text { quadricauda }\end{array}$} & \multirow{4}{*}{$\begin{array}{l}\text { Domestic wastewater collected from the } \\
\text { sewage wastewater treatment plant }\end{array}$} & BOD & $\begin{array}{l}\text { BOD = } \\
89.21 \%\end{array}$ & \\
\hline & & COD & $\begin{array}{l}\mathrm{COD}= \\
70.97 \%\end{array}$ & Kshirsagar, \\
\hline & & $\mathrm{NO}_{3}^{-}$ & $\begin{array}{l}\mathrm{NO}_{3}{ }^{-}= \\
70.32 \%\end{array}$ & (2013) \\
\hline & & $\mathrm{PO}_{4}^{3-}$ & $\begin{array}{l}\mathrm{PO}_{4}^{3-}= \\
81.34 \%\end{array}$ & \\
\hline & $\begin{array}{l}\text { Municipal wastewater collected from the } \\
\text { secondary treatment plant }\end{array}$ & $\mathrm{TP}$ & $\mathrm{TP}=11.40 \%$ & $\begin{array}{l}\text { Aravantinou et } \\
\text { al., (2013) }\end{array}$ \\
\hline Scenedesmus & & $\mathrm{NO}_{3}^{-}$ & $\mathrm{NO}_{3}{ }^{-}=99 \%$ & \\
\hline rubescens & Sewage wastewater collected from the & $\mathrm{NO}_{2}^{-}$ & $\mathrm{NO}_{2}^{-}=99 \%$ & \\
\hline & $2^{\text {nd }}$ clarifier treatment plant & $\mathrm{NH}_{4}^{+}$ & $\mathrm{NH}_{4}^{+}=99 \%$ & Su et al., (2012) \\
\hline & & $\mathrm{PO}_{4}^{3-}$ & $\mathrm{PO}_{4}^{3-}=99 \%$ & \\
\hline Scenedesmus & POME & BOD & $\mathrm{BOD}=$ & Rajkumar and \\
\hline
\end{tabular}




\begin{tabular}{|c|c|c|c|c|}
\hline $\begin{array}{l}\text { Microalgae } \\
\text { species }\end{array}$ & Source of the wastewater (ww) & Parameter & $\begin{array}{c}\text { Removal } \\
\text { efficiency } \\
(\%)\end{array}$ & Ref. \\
\hline \multirow[t]{5}{*}{ dimorphus } & & & $71.50 \%$ & \multirow[t]{5}{*}{ Takriff, (2015) } \\
\hline & & COD & $\mathrm{COD}=79 \%$ & \\
\hline & & TN & $\mathrm{TN}=87.50 \%$ & \\
\hline & & $\mathrm{NH}_{4}$ & $\begin{array}{c}\mathrm{NH}_{4}= \\
88.50 \%\end{array}$ & \\
\hline & & $\mathrm{TP}$ & $\mathrm{TP}=92.50 \%$ & \\
\hline \multirow{5}{*}{$\begin{array}{l}\text { Chlamydomonas } \\
\text { incerta }\end{array}$} & \multirow{5}{*}{ POME } & COD & $\mathrm{COD}=3.8 \%$ & \multirow{4}{*}{$\begin{array}{l}\text { Kamyab et al., } \\
\quad(2017)\end{array}$} \\
\hline & & $\mathrm{NO}_{3}{ }^{-}$ & $\mathrm{NO}_{3}{ }^{-}=12.5 \%$ & \\
\hline & & $\mathrm{NH}_{3}{ }^{-}$ & $\mathrm{NH}_{3}{ }^{-}=3.7 \%$ & \\
\hline & & $\mathrm{PO}_{4}$ & $\mathrm{PO}_{4}=70 \%$ & \\
\hline & & COD & $\begin{array}{l}\mathrm{COD}= \\
67.35 \%\end{array}$ & $\begin{array}{c}\text { Kamyab et al., } \\
(2015)\end{array}$ \\
\hline \multirow{2}{*}{$\begin{array}{l}\text { Chlamydomonas } \\
\text { mexicana }\end{array}$} & \multirow{2}{*}{ Treated piggery wastewater } & $\mathrm{TN}$ & $\mathrm{TN}=62 \%$ & \multirow{2}{*}{$\begin{array}{c}\text { Abou-Shanab et } \\
\text { al., (2013) }\end{array}$} \\
\hline & & $\mathrm{TP}$ & $\mathrm{TP}=30 \%$ & \\
\hline \multirow{4}{*}{$\begin{array}{l}\text { Chlamydomonas } \\
\text { polypyrenoideum }\end{array}$} & \multirow{4}{*}{$\begin{array}{l}\text { Dairy wastewater collected from the } \\
\text { oxidation pond }\end{array}$} & $\mathrm{NO}_{3}^{-}$ & $\mathrm{NO}_{3}{ }^{-}=90 \%$ & \multirow{4}{*}{$\begin{array}{l}\text { Kothari et al., } \\
\text { (2013) }\end{array}$} \\
\hline & & $\mathrm{NO}_{2}^{-}$ & $\mathrm{NO}_{2}^{-}=74 \%$ & \\
\hline & & $\mathrm{NH}_{4}^{+}$ & $\mathrm{NH}_{4}^{+}=90 \%$ & \\
\hline & & $\mathrm{PO}_{4}^{3-}$ & $\mathrm{PO}_{4}^{3-}=70 \%$ & \\
\hline \multirow{3}{*}{$\begin{array}{l}\text { Chlamydomonas } \\
\text { reinhardtii }\end{array}$} & \multirow{3}{*}{$\begin{array}{l}\text { Sewage wastewater collected from the } \\
2^{\text {nd }} \text { clarifier treatment plant }\end{array}$} & $\mathrm{NO}_{3}^{-}$ & $\mathrm{NO}_{3}{ }^{-}=99 \%$ & \multirow{3}{*}{ Su et al., (2012) } \\
\hline & & $\mathrm{NO}_{2}^{-}$ & $\mathrm{NO}_{2}{ }^{-}=99 \%$ & \\
\hline & & $\mathrm{NH}_{4}^{+}$ & $\mathrm{NH}_{4}{ }^{+}=99 \%$ & \\
\hline \multirow{2}{*}{$\begin{array}{l}\text { Chlamydomonas } \\
\text { sp. }\end{array}$} & \multirow{2}{*}{ Leachates } & $\mathrm{NH}_{4}^{+}$ & $\mathrm{NH}_{4}^{+}=70 \%$ & $\begin{array}{c}\text { Paskuliakova et } \\
\text { al., (2018a) }\end{array}$ \\
\hline & & $\mathrm{NH}_{4}^{+}$ & $\mathrm{NH}_{4}^{+}=83 \%$ & $\begin{array}{l}\text { Paskuliakova et } \\
\text { al., (2018b) }\end{array}$ \\
\hline \multirow{8}{*}{ Botryococcus sp. } & \multirow{4}{*}{$\begin{array}{l}\text { Diary wastewater collected from the goat } \\
\text { breeding farm }\end{array}$} & BOD & $\begin{array}{l}\text { BOD = } \\
73.30 \%\end{array}$ & \multirow{4}{*}{$\begin{array}{l}\text { Gani et al., } \\
\quad(2015 \mathrm{c})\end{array}$} \\
\hline & & COD & $\begin{array}{l}\mathrm{COD}= \\
48.80 \%\end{array}$ & \\
\hline & & $\mathrm{TN}$ & $\mathrm{TN}=48.28 \%$ & \\
\hline & & $\mathrm{PO}_{4}^{3-}$ & $\begin{array}{l}\mathrm{PO}_{4}^{3-}= \\
62.71 \%\end{array}$ & \\
\hline & \multirow{4}{*}{ Greywater from the residential area } & $\mathrm{BOD}$ & $\mathrm{BOD}=82 \%$ & \multirow{4}{*}{$\begin{array}{l}\text { Gani et al., } \\
\text { (2015b) }\end{array}$} \\
\hline & & $\mathrm{COD}$ & $\mathrm{COD}=88 \%$ & \\
\hline & & $\mathrm{TN}$ & $\mathrm{TN}=52 \%$ & \\
\hline & & $\mathrm{PO}_{4}{ }^{3-}$ & $\begin{array}{l}\mathrm{PO}_{4}{ }^{3-}= \\
37.50 \%\end{array}$ & \\
\hline \multirow{10}{*}{$\begin{array}{l}\text { Botryococcus } \\
\text { braunii }\end{array}$} & \multirow{3}{*}{$\begin{array}{c}\text { Domestic wastewater collected from the } \\
\text { Adyar river }\end{array}$} & BOD & $\begin{array}{l}\text { BOD = } \\
66.67 \%\end{array}$ & \multirow{3}{*}{ Raj, (2015) } \\
\hline & & COD & $\begin{array}{l}\text { COD = } \\
71.21 \%\end{array}$ & \\
\hline & & $\mathrm{NH}_{4}^{+}$ & $\begin{array}{l}\mathrm{NH}_{4}^{+}= \\
82.94 \%\end{array}$ & \\
\hline & \multirow{5}{*}{ Greywater collected from the hostel } & $\mathrm{TP}$ & $\mathrm{TP}=97.59 \%$ & \multirow{5}{*}{$\begin{array}{l}\text { Gokulan et al., } \\
\quad(2013)\end{array}$} \\
\hline & & BOD & $\begin{array}{l}\mathrm{BOD}= \\
76.13 \%\end{array}$ & \\
\hline & & COD & $\begin{array}{l}\text { COD = } \\
91.32 \% \\
\end{array}$ & \\
\hline & & $\mathrm{NO}_{3}^{-}$ & $\begin{array}{l}\mathrm{NO}_{3}^{-}= \\
69.58 \% \\
\end{array}$ & \\
\hline & & $\mathrm{NH}_{4}^{+}$ & $\begin{array}{l}\mathrm{NH}_{4}^{+}= \\
97.82 \%\end{array}$ & \\
\hline & \multirow{2}{*}{$\begin{array}{l}\text { Municipal wastewater collected from the } \\
\text { primary settling tank }\end{array}$} & $\mathrm{NO}_{3}^{-}$ & $\begin{array}{l}\mathrm{NO}_{3}^{-}= \\
60.31 \%\end{array}$ & Can et al., \\
\hline & & $\mathrm{NO}_{2}^{-}$ & $\begin{array}{l}\mathrm{NO}_{2}^{-}= \\
53.50 \%\end{array}$ & (2013) \\
\hline
\end{tabular}




\begin{tabular}{|c|c|c|c|c|}
\hline $\begin{array}{l}\text { Microalgae } \\
\text { species }\end{array}$ & Source of the wastewater (ww) & Parameter & $\begin{array}{c}\text { Removal } \\
\text { efficiency } \\
(\%)\end{array}$ & Ref. \\
\hline \multirow{4}{*}{ Oscillatoria sp. } & & $\mathrm{NH}_{4}^{+}$ & $\mathrm{NH}_{4}{ }^{+}=100 \%$ & \\
\hline & & $\mathrm{PO}_{4}{ }^{3-}$ & $\mathrm{PO}_{4}{ }^{3-}=99 \%$ & \\
\hline & \multirow{4}{*}{$\begin{array}{l}\text { Municipal wastewater collected from the } \\
\text { Pune Corporation }\end{array}$} & $\mathrm{NO}_{3}^{-}$ & $\mathrm{NO}_{3}{ }^{-}=97 \%$ & \multirow{4}{*}{$\begin{array}{l}\text { Azarpira et al., } \\
\quad(2014)\end{array}$} \\
\hline & & $\mathrm{PO}_{4}^{3-}$ & $\mathrm{PO}_{4}^{3-}=93 \%$ & \\
\hline \multirow{2}{*}{ Nostoc commune } & & $\mathrm{NO}_{3}^{-}$ & $\mathrm{NO}_{3}{ }^{-}=96 \%$ & \\
\hline & & $\mathrm{PO}_{4}^{3-}$ & $\mathrm{PO}_{4}{ }^{3-}=84 \%$ & \\
\hline \multirow{4}{*}{ Nostoc sp. } & \multirow[t]{2}{*}{ Primary treated domestic wastewater } & $\mathrm{NO}_{3}^{-}$ & $\begin{array}{l}\mathrm{NO}_{3}^{-}= \\
45.68 \%\end{array}$ & \multirow{2}{*}{$\begin{array}{l}\text { Sharma and } \\
\text { Khan, (2013) }\end{array}$} \\
\hline & & $\mathrm{NH}_{4}^{+}$ & $\mathrm{NH}_{4}^{+}=90 \%$ & \\
\hline & \multirow{2}{*}{$\begin{array}{l}\text { Dairy wastewater collected from the } \\
\text { treatment plant }\end{array}$} & BOD & $\begin{array}{l}\mathrm{BOD}= \\
40.44 \%\end{array}$ & \multirow{2}{*}{$\begin{array}{l}\text { Kotteswari et } \\
\text { al., (2012) }\end{array}$} \\
\hline & & $\mathrm{PO}_{4}^{3-}$ & $\begin{array}{l}\mathrm{PO}_{4}{ }^{3-}= \\
21.08 \%\end{array}$ & \\
\hline \multirow{3}{*}{$\begin{array}{l}\text { Desmodesmus } \\
\text { sp. }\end{array}$} & \multirow{3}{*}{$\begin{array}{l}\text { Sewage wastewater collected from the } \\
\text { facultative lagoon treatment plant } \\
\text { Synthetic industrial wastewater }\end{array}$} & $\mathrm{TN}$ & $\mathrm{TN}=80 \%$ & \multirow{2}{*}{$\begin{array}{l}\text { Komolafe et al., } \\
\text { (2014) }\end{array}$} \\
\hline & & $\mathrm{PO}_{4}^{3-}$ & $\begin{array}{l}\mathrm{PO}_{4}^{3-}= \\
38.70 \%\end{array}$ & \\
\hline & & $\mathrm{TP}$ & $\mathrm{TP}=94 \%$ & $\begin{array}{l}\text { Rugnini et al., } \\
(2018)\end{array}$ \\
\hline \multirow{2}{*}{$\begin{array}{l}\text { Tetraselmis } \\
\text { suecica }\end{array}$} & \multirow{2}{*}{$\begin{array}{l}\text { Aquaculture wastewater collected from } \\
\text { the fish farm }\end{array}$} & $\mathrm{TN}$ & $\mathrm{TN}=95.70 \%$ & \multirow{2}{*}{$\begin{array}{l}\text { Michels et al., } \\
\text { (2014) }\end{array}$} \\
\hline & & TP & $\mathrm{TP}=99.70 \%$ & \\
\hline \multirow{3}{*}{ Tetraselmis chuii } & \multirow{3}{*}{$\begin{array}{c}\text { Aquaculture }- \text { recirculation aquaculture } \\
\text { system (RAS) }\end{array}$} & $\mathrm{TN}$ & $\mathrm{TN}=69.50 \%$ & \multirow{3}{*}{$\begin{array}{l}\text { Sirakov and } \\
\text { Velichkova, } \\
\text { (2014) }\end{array}$} \\
\hline & & $\mathrm{NO}_{2}^{-}$ & $\begin{array}{l}\mathrm{NO}_{2}^{-}= \\
79.17 \%\end{array}$ & \\
\hline & & $\mathrm{PO}_{4}^{3-}$ & $\begin{array}{l}\mathrm{PO}_{4}^{3-}= \\
64.70 \%\end{array}$ & \\
\hline $\begin{array}{l}\text { Neochloris } \\
\text { vigensis }\end{array}$ & \multirow{2}{*}{$\begin{array}{l}\text { Municipal wastewater collected from the } \\
\text { secondary treatment plant }\end{array}$} & TP & $\mathrm{TP}=53.40 \%$ & \multirow{2}{*}{$\begin{array}{l}\text { Aravantinou et } \\
\text { al., (2013) }\end{array}$} \\
\hline $\begin{array}{l}\text { Chlorococcum } \\
\text { spec. }\end{array}$ & & $\mathrm{TP}$ & $\mathrm{TP}=25.10 \%$ & \\
\hline \multirow{10}{*}{ Spirulina sp. } & \multirow{4}{*}{ Synthetic dairy wastewater } & BOD & $\mathrm{BOD}=81 \%$ & \multirow{4}{*}{$\begin{array}{l}\text { Sumithrabhai et } \\
\text { al., (2016) }\end{array}$} \\
\hline & & COD & $\mathrm{COD}=83 \%$ & \\
\hline & & $\mathrm{TN}$ & $\mathrm{TN}=77 \%$ & \\
\hline & & $\mathrm{TP}$ & $\mathrm{TP}=69 \%$ & \\
\hline & & COD & $\mathrm{COD}=77 \%$ & \\
\hline & $\begin{array}{c}\text { Dairy wastewater collected from the } \\
\text { factory }\end{array}$ & $\mathrm{NO}_{3}^{-}$ & $\mathrm{NO}_{3}{ }^{-}=80 \%$ & Ahmed, (2014) \\
\hline & & $\mathrm{PO}_{4}^{3-}$ & $\mathrm{PO}_{4}{ }^{3-}=72 \%$ & \\
\hline & \multirow{3}{*}{$\begin{array}{l}\text { POME collected from the anaerobic } \\
\text { fourth pond }\end{array}$} & COD & $\mathrm{COD}=50 \%$ & \\
\hline & & $\mathrm{TN}$ & $\mathrm{TN}=40 \%$ & $\begin{array}{l}\text { Hadiyanto et } \\
\text { al., (2014) }\end{array}$ \\
\hline & & $\mathrm{TP}$ & $\mathrm{TP}=40 \%$ & \\
\hline \multirow{8}{*}{$\begin{array}{l}\text { Spirulina } \\
\text { platensis }\end{array}$} & \multirow{3}{*}{$\begin{array}{l}\text { POME collected from the anaerobic } \\
\text { fourth pond }\end{array}$} & COD & $\begin{array}{l}\mathrm{COD}= \\
50.79 \% \\
\end{array}$ & \multirow{3}{*}{$\begin{array}{l}\text { Hadiyanto et } \\
\text { al., (2013) }\end{array}$} \\
\hline & & $\mathrm{TN}$ & $\mathrm{TN}=96.50 \%$ & \\
\hline & & $\mathrm{TP}$ & $\mathrm{TP}=85.92 \%$ & \\
\hline & & BOD & $\begin{array}{l}\mathrm{BOD}= \\
78.30 \%\end{array}$ & \\
\hline & & COD & $\begin{array}{l}\mathrm{COD}= \\
84.90 \%\end{array}$ & Rajkumar and \\
\hline & POME & $\mathrm{TN}$ & $\mathrm{TN}=91 \%$ & Takriff, (2015) \\
\hline & & $\mathrm{NH}_{4}-\mathrm{N}$ & $\begin{array}{l}\mathrm{NH}_{4}-\mathrm{N}= \\
93.80 \% \\
\end{array}$ & \\
\hline & & $\mathrm{TP}$ & $\mathrm{TP}=96.80 \%$ & \\
\hline $\begin{array}{l}\text { Auxenochlorella } \\
\text { protothecoides }\end{array}$ & $\begin{array}{c}\text { Municipal wastewater collected from the } \\
\text { treatment plant }\end{array}$ & COD & $\begin{array}{l}\mathrm{COD}= \\
88.99 \%\end{array}$ & $\begin{array}{l}\text { Zhou et al., } \\
\quad(2012)\end{array}$ \\
\hline
\end{tabular}




\begin{tabular}{|c|c|c|c|c|}
\hline $\begin{array}{l}\text { Microalgae } \\
\text { species }\end{array}$ & Source of the wastewater (ww) & Parameter & $\begin{array}{c}\begin{array}{c}\text { Removal } \\
\text { efficiency } \\
(\%)\end{array} \\
\end{array}$ & Ref. \\
\hline & & $\mathrm{TN}$ & $\mathrm{TN}=59.70 \%$ & \\
\hline & & $\mathrm{TP}$ & $\mathrm{TP}=81.52 \%$ & \\
\hline \multirow{3}{*}{ Oocystis sp. } & \multirow{3}{*}{$\begin{array}{l}\text { Fish processing wastewater collected } \\
\text { from the fish farm }\end{array}$} & COD & $\begin{array}{l}\mathrm{COD}= \\
71.10 \%\end{array}$ & \multirow{3}{*}{$\begin{array}{l}\text { Riano et al., } \\
\quad(2011)\end{array}$} \\
\hline & & $\mathrm{NH}_{4}^{+}$ & $\mathrm{NH}_{4}^{+}=95 \%$ & \\
\hline & & $\mathrm{TP}$ & $\mathrm{TP}=74.10$ & \\
\hline Euglena viridis & \multirow{5}{*}{$\begin{array}{l}\text { Sewage wastewater collected from the } \\
\text { drain opens into river, Yamuna }\end{array}$} & BOD & $\begin{array}{l}\text { BOD = } \\
96.20 \%\end{array}$ & \multirow{5}{*}{$\begin{array}{l}\text { Sengar et al., } \\
\quad(2011)\end{array}$} \\
\hline $\begin{array}{l}\text { Gloeocapsa } \\
\text { gelatinosa }\end{array}$ & & COD & $\mathrm{COD}=82 \%$ & \\
\hline \multirow{3}{*}{ Synedra affinis } & & $\mathrm{NO}_{3}^{-}$ & $\mathrm{NO}_{3}{ }^{-}=100 \%$ & \\
\hline & & $\mathrm{NO}_{2}^{-}$ & $\mathrm{NO}_{2}^{-}=100 \%$ & \\
\hline & & $\mathrm{PO}_{4}^{3-}$ & $\mathrm{PO}_{4}^{3-}=100 \%$ & \\
\hline \multirow{6}{*}{$\begin{array}{l}\text { Synechocystis } \\
\text { salina }\end{array}$} & \multirow{6}{*}{$\begin{array}{l}\text { Chemical (based products) wastewater } \\
\text { collected from the Periyor }\end{array}$} & $\mathrm{NO}_{3}^{-}$ & $\begin{array}{l}\mathrm{NO}_{3}^{-}= \\
80.90 \%\end{array}$ & \multirow{6}{*}{$\begin{array}{l}\text { Dominic et al., } \\
\text { (2009) }\end{array}$} \\
\hline & & $\mathrm{NO}_{2}^{-}$ & $\mathrm{NO}_{2}^{-}=100 \%$ & \\
\hline & & $\mathrm{PO}_{4}^{3-}$ & $\mathrm{PO}_{4}^{3-}=75 \%$ & \\
\hline & & $\mathrm{NO}_{3}{ }^{-}$ & $\begin{array}{l}\mathrm{NO}_{3}^{-}= \\
82.50 \%\end{array}$ & \\
\hline & & $\mathrm{NO}_{2}^{-}$ & $\begin{array}{l}\mathrm{NO}_{2}{ }^{-}= \\
96.23 \%\end{array}$ & \\
\hline & & $\mathrm{PO}_{4}^{3-}$ & $\mathrm{PO}_{4}{ }^{3-}=64.52$ & \\
\hline \multirow{4}{*}{ Pithopora sp. } & \multirow{4}{*}{$\begin{array}{c}\text { Thermal wastewater collected from the } \\
\text { power station }\end{array}$} & BOD & $\begin{array}{l}\text { BOD = } \\
88.23 \%\end{array}$ & \multirow{4}{*}{$\begin{array}{c}\text { Murugesan and } \\
\text { Dhamotharan, } \\
\text { (2009) }\end{array}$} \\
\hline & & COD & $\begin{array}{l}\text { COD = } \\
87.75 \%\end{array}$ & \\
\hline & & $\mathrm{NO}_{3}^{-}$ & $\begin{array}{l}\mathrm{NO}_{3}^{-}= \\
23.07 \%\end{array}$ & \\
\hline & & $\mathrm{PO}_{4}^{3-}$ & $\begin{array}{l}\mathrm{PO}_{4}^{3-}= \\
89.37 \%\end{array}$ & \\
\hline \multirow{3}{*}{$\begin{array}{l}\text { Nannochloris } \\
\text { oculata }\end{array}$} & \multirow{3}{*}{$\begin{array}{c}\text { Aquaculture wastewater -recirculation } \\
\text { aquaculture system (RAS) }\end{array}$} & TN & $\mathrm{TN}=78.40 \%$ & \multirow{3}{*}{$\begin{array}{l}\text { Sirakov and } \\
\text { Velichkova, } \\
\text { (2014) }\end{array}$} \\
\hline & & $\mathrm{NO}_{2}^{-}$ & $\begin{array}{l}\mathrm{NO}_{2}^{-}= \\
84.38 \%\end{array}$ & \\
\hline & & $\mathrm{PO}_{4}^{3-}$ & $\begin{array}{l}\mathrm{PO}_{4}^{3-}= \\
14.70 \%\end{array}$ & \\
\hline \multirow{6}{*}{ Characium sp. } & \multirow{6}{*}{$\begin{array}{l}\text { POME collected from the anaerobic } \\
\text { pond }\end{array}$} & COD & $\begin{array}{l}\mathrm{COD}= \\
45.41 \% \\
\end{array}$ & \multirow{6}{*}{$\begin{array}{l}\text { Selvam et al., } \\
\qquad(2015)\end{array}$} \\
\hline & & $\mathrm{TN}$ & $\mathrm{TN}=88.60 \%$ & \\
\hline & & $\mathrm{NH}_{3}^{-}$ & $\begin{array}{l}\mathrm{NH}_{3}^{-}= \\
90.35 \%\end{array}$ & \\
\hline & & $\mathrm{NH}_{4}{ }^{+}$ & $\mathrm{NH}_{4}{ }^{+}=87 \%$ & \\
\hline & & $\mathrm{TP}$ & $\mathrm{TP}=99.5 .0 \%$ & \\
\hline & & $\mathrm{PO}_{4}^{3-}$ & $\begin{array}{l}\mathrm{PO}_{4}{ }^{3-}= \\
99.10 \%\end{array}$ & \\
\hline \multirow{4}{*}{$\begin{array}{l}\text { Micro algal } \\
\text { mixture }\end{array}$} & POME collected from the final pond & COD & $\begin{array}{l}\mathrm{COD}= \\
71.16 \%\end{array}$ & $\begin{array}{c}\text { Kamyab et al., } \\
(2014)\end{array}$ \\
\hline & \multirow{2}{*}{ Textile wastewater } & $\mathrm{TN}$ & $\mathrm{TN}=70.10 \%$ & \multirow{2}{*}{$\begin{array}{l}\text { Huy et al., } \\
\text { (2018) }\end{array}$} \\
\hline & & $\mathrm{TP}$ & $\mathrm{TP}=100 \%$ & \\
\hline & Urban wastewater & $\begin{array}{l}\text { BOD } \\
\text { COD } \\
\text { TN } \\
\text { TP } \\
\end{array}$ & $\begin{array}{c}\mathrm{BOD}=51 \% \\
\mathrm{COD}=91 \% \\
\mathrm{TN}=95.10 \% \\
\mathrm{TP}=88.9 \%\end{array}$ & $\begin{array}{l}\text { Marella et al., } \\
\quad(2018)\end{array}$ \\
\hline \multirow{3}{*}{$\begin{array}{l}\text { Algal-bacterial } \\
\text { culture }\end{array}$} & \multirow{3}{*}{$\begin{array}{l}\text { Municipal wastewater collected from the } \\
2^{\text {nd }} \text { clarifier treatment plant }\end{array}$} & COD & $\mathrm{COD}=98.2 \%$ & \multirow{3}{*}{ Su et al., (2011) } \\
\hline & & TKN & $\mathrm{TKN}=88.3 \%$ & \\
\hline & & $\mathrm{PO}_{4}^{3-}$ & $\begin{array}{c}\mathrm{PO}_{4}^{3-}= \\
64.8 \%\end{array}$ & \\
\hline
\end{tabular}




\begin{tabular}{|c|c|c|c|c|}
\hline $\begin{array}{l}\text { Microalgae } \\
\text { species }\end{array}$ & Source of the wastewater (ww) & Parameter & $\begin{array}{c}\begin{array}{c}\text { Removal } \\
\text { efficiency } \\
(\%)\end{array} \\
\end{array}$ & Ref. \\
\hline & \multirow{3}{*}{ Synthetic wastewater } & COD & $\begin{array}{l}\mathrm{COD}= \\
65.62 \%\end{array}$ & \multirow{3}{*}{ Ji et al., (2018) } \\
\hline & & TN & $\mathrm{TN}=21.56 \%$ & \\
\hline & & $\mathrm{TP}$ & $\mathrm{TP}=70.82 \%$ & \\
\hline & \multirow[b]{2}{*}{ Municipal wastewater } & $\mathrm{TN}$ & $\mathrm{TN}=83 \%$ & \multirow{2}{*}{$\begin{array}{c}\text { Delgadillo- } \\
\text { Mirquez et al., } \\
(2016) \\
\end{array}$} \\
\hline & & $\mathrm{TP}$ & $\mathrm{TP}=100 \%$ & \\
\hline Algal biofilm & Artificial municipal wastewater & $\mathrm{TP}$ & $\mathrm{TP}=97 \%$ & $\begin{array}{c}\text { Sukačova et al., } \\
(2015)\end{array}$ \\
\hline
\end{tabular}

\section{Application of phycoremediation in treating wastewater using suspended-free cells of microalgae}

Microorganisms especially microalgae have received significant attention in wastewater treatment. This is due to their capability to take up and assimilate plant nutrients, pesticides, organic and inorganic pollutants in their unicellular structure (Sahu, 2014). According to Pittman et al. (2011), a lot of microalgae species thrive in wastewater containing high concentration of nitrogen and phosphorus and use them as a vital source of energy for their growth. This leads to significant uptake and reduction of nutrient concentrations.

Cultivation of microalgae in wastewater treatment system offers several advantages. The process is simple, economical and sustainable. Zhou et al. (2012) reported that growing microalgae in wastewater is probably the most promising approach to reduce costs of production in term of nutrients and clean water supply. Furthermore, Rawat et al. (2011) reported that readily available municipal wastewater can be used as a growth media to cultivate microalgae together with the added benefits of bioremediation (e.g. phycoremediation).

Chlorella sp. reduced COD (15\%), total nitrogen (TN) $(91 \%)$, ammonium $\left(\mathrm{NH}_{4}^{+}\right)$ $(100 \%)$ and total phosphorus (TP) (93\%) from synthetic aquaculture for 22 days (Komolafe et al., 2014). It has been shown that $C$. vulgaris grown in sewage wastewater accomplished removal of BOD (98.70\%), COD (98.30\%), total kjeldahl nitrogen (TKN) (93.10\%), nitrate $\left(\mathrm{NO}_{3}{ }^{-}\right)(98.30 \%)$, TP $(98 \%)$ and (phosphate) $\mathrm{PO}_{4}{ }^{3-}(98.60 \%)$ (Wang et al., 2010). As Whangchenchom et al., (2014) reported that Scenedesmus sp. was capable of removing $73.37 \%$ of COD from wastewaters in Thailand. Suspended free-cell of Scenedesmus sp., cultivated in synthetic $2 \mathrm{f}$ medium reduced $\mathrm{NO}_{3}{ }^{-}$and $\mathrm{PO}_{4}{ }^{3-}$ by $20 \%$ and $30 \%$, respectively (Fierro et al., 2008). Removal of $\mathrm{TN}, \mathrm{NO}_{3}^{-}, \mathrm{NH}_{4}{ }^{+}$, and $\mathrm{TP}$ from piggery wastewater using Scenedesmus obliquus was achieved at 60, 84, 57 and $83 \%$, respectively (Ji et al., 2013). Sacristán de Alva et al., (2013) and Su et al. (2012) used others strains of Scenedesmus species to treat wastewater. High removal of $\mathrm{NO}_{3}{ }^{-}$, (nitrite) $\mathrm{NO}_{2}{ }^{-}, \mathrm{NH}_{4}{ }^{+}$, and $\mathrm{PO}_{4}{ }^{3-}$ were achieved as compared to Kothari et al. (2013) using Chlamydomonas polypyrenoideum. Gokulan et al. (2013) evaluated the nutrients removal efficiency of Botryococcus braunii in greywater samples collected from a hostel. They reported that B. braunii removed $76.13 \%, 91.32 \%, 69.58 \%, 97.82 \%$, $97.59 \%$ of BOD, $\mathrm{COD}, \mathrm{NO}_{3}{ }^{-}, \mathrm{NH}_{4}{ }^{+}$and TP, respectively. Ganeshkumar et al. (2018) studied the potential of Chlorella $s p$. to treat mixed wastewater from piggery and winery in India. The treatment process was conducted for 10 days in at $23^{\circ} \mathrm{C}$ in an orbital shaker. The initial concentration of TN and TP of $284 \mathrm{mg} / \mathrm{L}$ and $11 \mathrm{mg} / \mathrm{L}$ was 
reduced to $30.22 \mathrm{mg} / \mathrm{L}$ and $4.78 \mathrm{mg} / \mathrm{L}$, respectively. Therefore, the study achieved good removal of TN and TP up to $89.36 \%$ and $56.56 \%$, respectively. The removal efficiency of nutrients, COD and BOD from various wastewaters for other studies were summarized in Table 2.

It can be summarized that the phycoremediation technology has the ability to remove nutrients from wastewaters up to certain removal efficiency. However, the removal efficiency of nutrients depends on the types of wastewaters and microalgae species to be treated and be used as bioremediation agent, respectively. Besides that, some microalgae species shown great removal efficiency of nitrogen removal from wastewaters. An explanation for this was due to the role of nitrogen to build microalgae cells through anabolism pathway. Protein, chlorophyll, amino acids and also genetic materials are major made up of nitrogen (McElwee et al., 2006). Thus, the phycoremediation process has a potential to reduce the nitrogen to the lowest concentration from wastewaters.

Rock minerals, soil erosion and animal waste decomposition are the natural sources of phosphorus in the aquatic system. Phosphorus removal from wastewaters is vital to avoid eutrophication problem. This problem could be achieved through phycoremediation process which exhibited great removal efficiency of phosphorus as shown in Table 2. Table 2 also shows the phycoremediation process for various wastewaters using different types of microalgae species.

\section{Algae immobilization}

De-Bashan and Bashan (2010) defined immobilized cells as living cells by which natural or artificial methods have been used to restrict independent movement from its original position to all part of an aqueous phase. Immobilization of microalgae in polymers can overcome problems associated with biomass harvesting from suspended free-cells cultivated in wastewater. Although solid-liquid separation technologies such as centrifugation and filtration can be used to separate free cells. There are several types of immobilization: (i) covalent coupling (ii) affinity immobilization (iii) adsorption (iv) confinement in liquid-liquid emulsion (v) capture behind semi-permeable membrane; and (vi) entrapment in polymers (Malik, 2002; Eroglu et al., 2015). They can be further categorized into "passive" (immobilization onto natural or synthetic gel-like carriers) and "active" (using flocculants, chemical attachment, and gel encapsulation) (MorenoGarrido, 2008).

Immobilization of microalgae by entrapment using gel polymers is the most common method in wastewater treatment applications (Eroglu et al., 2015). There is a physical separation between the microorganisms and the treated wastewater in polymeric immobilization, and this is similar to biofiltration. The microalgae cells are immobilized and entrapped alive in the polymer gel matrix. The gel pores are smaller in size than the microalgae. The wastewater fluid flows through the pores of the polymer and sustains microalgae metabolism and growth (Cohen, 2001). The wastewater diffuses through the polymer pores, resulting in uptake of nutrients by the entrapped microalgae cells. Compared to suspended free-cells microalgae cultures cells, the following are some advantages of immobilized microalgae in treating wastewater: (i) provides stability to the photobioreactor (PBR) system design (ii) enhance operational stability (iii) easy to regenerate immobilized microalgae (iv) avoids cell washout (v) facilitates the cultivation of microalgae and easy of harvesting of their biomass (vi) high and rapid 
uptake of nutrient plus shorter retention time (vii) allows bioprocess with better light utilization efficiency per area and higher cell densities (viii) yields significant metabolite concentrations (ix) high tolerance against harsh environments like extreme $\mathrm{pH}$, temperature, ultraviolet radiation and toxic compounds $(\mathrm{x})$ protects aging cultures against the harmful effects of photoinhibition (xi) rotects microalgae cells from being consumed by wild zooplankton (xii) enhances the capacity of biosorption and bioactivity of the biomass; and (xiii) allow immobilization of more than one microorganism (usually microalgae co-immobilized with bacteria species) (de-Bashan and Bashan, 2010; Eroglu et al., 2015; Vasilieva et al., 2016).

Successful entrapment allows microalgae cells to move freely within the space of beads with the optimal pore size that facilitates diffusion of wastewater and metabolic products into and/or out of the polymer system (Malik, 2002). According to Eroglu et al. (2015), the dual effect of enhanced photosynthetic rate and ionic exchange between the nutrient ions and the immobilized matrix results in efficient removal of nutrients from wastewater. Anionic gels (such as carrageenan and alginate) and cationic gels (such as chitosan) adsorb cations (e.g. $\left.\mathrm{NH}_{4}{ }^{+}\right)$and anions $\left(\mathrm{PO}_{4}{ }^{3-}, \mathrm{NO}_{3}{ }^{-}, \mathrm{NO}_{2}{ }^{-}\right)$with high efficiency. In addition, $\mathrm{PO}_{4}{ }^{3-}$ is removed efficiently from wastewater via precipitation by calcium ions of alginate or chitosan gels.

Immobilizing materials or carriers can be put into two categories; synthetic and natural polymer (Eroglu et al., 2015; Vasilieva et al., 2016). Examples of synthetic polymers for wastewater treatment include polyacrylamide, polyurethane, polyvinyl, polypropylene, and polystyrene, polysulfone, epoxy resins, and filter papers. Natural polymers can be derived from plant polysaccharides. These include agar, cellulose, alginate, carrageenan, and chitosan. The immobilizing materials possess hydrophilic properties for enhanced diffusion of wastewater into the beads.

Natural polymers such as alginate, carrageenan, and chitosan are the most commonly used immobilizing materials in wastewater treatment (Shi et al., 2007; Zhang et al., 2008; Moreno-Garrido, 2008; Eroglu et al., 2015; Sumithrabhai et al., 2016), and this due to the following advantages. They are (i) non-toxic, easy to process, and costeffective; (ii) transparent and permeable; (iii) hydrophilic and have higher nutrient/product diffusion rates than synthetic polymers; (iv) more environmentally friendly and produces less hazardous waste following treatment; and (v) bio-compatible. The use of these natural polymers in wastewater treatment also poses some disadvantages. They are (i) less stable as they dissolve slightly in highly contaminated wastewater; (ii) do not retain their polymeric structure in the presence of high concentration of phosphate and some cations (e.g. calcium and magnesium); and (iii) susceptible to microbial degradation. However, the degradation of the natural polymer in highly contaminated wastewater can be minimized by the composite assembly of polymers. For example, the stability of carrageenan gels can be enhanced by mixing the carrageenan with polyacrylamide (Eroglu et al., 2015).

There is a generic method for immobilizing microalgae onto polymers. Briefly, the microbial suspension is mixed with the macromolecular monomers of the selected polymer (e.g. alginate, carrageenan, chitosan solution) to form polymeric gels (e.g. spherical beads produced via the small orifice of syringe) after solidification. The monomers cross-link to each other with di- and multi-valent cations such as calcium chloride to produce polymers with entrapped microbes within the matrix. Generally, as the concentration of monomers and cross-linking agents' increases, the mechanical strength of the polymer increases, resulting in pore size reduction (de-Bashan and 
Bashan, 2010). Table 3 shows the removal efficiency of pollutants from wastewater using different immobilizing materials.

Table 3. Comparison of phycoremediation efficiency by immobilized algae grown in the various wastewaters

\begin{tabular}{|c|c|c|c|c|c|}
\hline $\begin{array}{l}\text { Microalgae } \\
\text { Species }\end{array}$ & $\begin{array}{c}\text { Immobilizing } \\
\text { material }\end{array}$ & Source of the wastewater & Parameter & $\begin{array}{c}\begin{array}{c}\text { Removal } \\
\text { efficiency } \\
(\%)\end{array} \\
\end{array}$ & Ref. \\
\hline \multirow{12}{*}{$\begin{array}{l}\text { Chlorella } \\
\text { vulgaris }\end{array}$} & $\begin{array}{c}\text { Chitosan } \\
\text { nanofiber } \\
\text { mats }\end{array}$ & Synthetic sewage effluent & $\mathrm{NO}_{3}^{-}$ & $\mathrm{NO}_{3}^{-}=87 \%$ & $\begin{array}{l}\text { Eroglu et } \\
\text { al., (2012) }\end{array}$ \\
\hline & \multirow{4}{*}{$\begin{array}{l}\text { Twin-layer } \\
\text { system }\end{array}$} & \multirow{3}{*}{$\begin{array}{l}\text { Synthetic secondary } \\
\text { wastewater }\end{array}$} & $\mathrm{NO}_{3}^{-}$ & $\mathrm{NO}_{3}^{-}=93 \%$ & \multirow{3}{*}{$\begin{array}{l}\text { Shi et al., } \\
\text { (2007) }\end{array}$} \\
\hline & & & $\mathrm{NH}_{4}^{+}$ & $\mathrm{NH}_{4}^{+}=94 \%$ & \\
\hline & & & $\mathrm{PO}_{4}{ }^{3-}$ & $\mathrm{PO}_{4}^{3-}=89 \%$ & \\
\hline & & Municipal wastewater & $\mathrm{NO}_{3}^{-}$ & $\mathrm{NO}_{3}^{-}=98 \%$ & $\begin{array}{l}\text { Shi et al., } \\
\text { (2007) }\end{array}$ \\
\hline & \multirow{5}{*}{$\begin{array}{c}\text { Calcium } \\
\text { alginate beads }\end{array}$} & \multirow{3}{*}{$\begin{array}{l}\text { Domestic primary treated } \\
\text { wastewater }\end{array}$} & $\mathrm{NO}_{3}^{-}$ & $\begin{array}{l}\mathrm{NO}_{3}^{-=} \\
96.40 \%\end{array}$ & \multirow{3}{*}{$\begin{array}{l}\text { Hameed, } \\
(2007)\end{array}$} \\
\hline & & & $\mathrm{NH}_{4}^{+}$ & $\mathrm{NH}_{4}^{+}=100 \%$ & \\
\hline & & & $\mathrm{PO}_{4}{ }^{3-}$ & $\mathrm{PO}_{4}^{3-}=95 \%$ & \\
\hline & & \multirow{2}{*}{$\begin{array}{l}\text { Synthetic primary settled } \\
\text { domestic wastewater }\end{array}$} & $\mathrm{NH}_{4}^{+}$ & $\mathrm{NH}_{4}^{+}=100 \%$ & \multirow{2}{*}{$\begin{array}{l}\text { Tam and } \\
\text { Wong, } \\
(2000)\end{array}$} \\
\hline & & & $\mathrm{PO}_{4}^{3-}$ & $\mathrm{PO}_{4}^{3-}=95 \%$ & \\
\hline & \multirow{2}{*}{$\begin{array}{l}\text { Carrageenan } \\
\text { beads }\end{array}$} & \multirow{2}{*}{$\begin{array}{l}\text { Sewage wastewater collected } \\
\text { from the primary settling tank }\end{array}$} & $\mathrm{NH}_{4}^{+}$ & $\mathrm{NH}_{4}^{+}=95 \%$ & \multirow{2}{*}{$\begin{array}{l}\text { Lau et al., } \\
\text { (1997) }\end{array}$} \\
\hline & & & $\mathrm{PO}_{4}{ }^{3-}$ & $\mathrm{PO}_{4}^{3-}=99 \%$ & \\
\hline \multirow{3}{*}{$\begin{array}{c}\text { Chlorella } \\
\text { vulgaris and } \\
\text { Azospirillum } \\
\text { brasilense) }\end{array}$} & \multirow[b]{4}{*}{$\begin{array}{l}\text { Alginate } \\
\text { beads }\end{array}$} & \multirow{4}{*}{$\begin{array}{c}\text { Municipal wastewater } \\
\text { collected from the stream of } \\
\text { wastewater after the initial } \\
\text { aerobic activated sludge } \\
\text { treatment }\end{array}$} & $\mathrm{NO}_{3}^{-}$ & $\mathrm{NO}_{3}{ }^{-}=15 \%$ & \multirow{3}{*}{$\begin{array}{c}\text { De-Bashan } \\
\text { et al., } \\
\text { (2004) }\end{array}$} \\
\hline & & & $\mathrm{NH}_{4}^{+}$ & $\mathrm{NH}_{4}{ }^{+}=100 \%$ & \\
\hline & & & $\mathrm{PO}_{4}^{3-}$ & $\mathrm{PO}_{4}^{3-}=36 \%$ & \\
\hline $\begin{array}{l}\text { Chlorella } \\
\text { sorokiniana } \\
\text { and } \\
\text { Azospirillum } \\
\text { brasilense }\end{array}$ & & & $\mathrm{PO}_{4}^{3-}$ & $\mathrm{PO}_{4}{ }^{3-}=72 \%$ & $\begin{array}{l}\text { Hernandez } \\
\text { et al., } \\
(2006)\end{array}$ \\
\hline \multirow{2}{*}{$\begin{array}{c}\text { Chlorella } \\
\text { pyrenoidosa } \\
\text { and activated } \\
\text { sludge }\end{array}$} & \multirow{2}{*}{$\begin{array}{l}\text { Polyvinyl } \\
\text { alcohol } \\
\text { (PVA) - } \\
\text { sulfate gel } \\
\end{array}$} & \multirow[b]{2}{*}{ Synthetic wastewater } & $\mathrm{NO}_{3}^{-}$ & $\mathrm{NO}_{3}{ }^{-}=80 \%$ & \multirow{2}{*}{$\begin{array}{l}\text { Huang and } \\
\text { Wang, } \\
\text { (2003) }\end{array}$} \\
\hline & & & $\mathrm{PO}_{4}^{3-}$ & $\mathrm{PO}_{4}^{-3}=88 \%$ & \\
\hline \multirow{3}{*}{$\begin{array}{l}\text { Chlorella } \\
\text { salina }\end{array}$} & \multirow{7}{*}{$\begin{array}{c}\text { Sodium } \\
\text { alginate beads }\end{array}$} & \multirow{3}{*}{ Tannery wastewater } & $\mathrm{NO}_{3}^{-}$ & $\begin{array}{l}\mathrm{NO}_{3}= \\
98.71 \%\end{array}$ & \multirow{3}{*}{$\begin{array}{l}\text { Jaysudha } \\
\text { and } \\
\text { Sampathku } \\
\text { mar, (2014) }\end{array}$} \\
\hline & & & $\mathrm{NH}_{4}^{+}$ & $\begin{array}{l}\mathrm{NH}_{4}= \\
98.54 \%\end{array}$ & \\
\hline & & & $\mathrm{PO}_{4}{ }^{3-}$ & $\mathrm{PO}_{4}=99.39 \%$ & \\
\hline \multirow{4}{*}{$\begin{array}{l}\text { Spirulina } \\
\text { maxima }\end{array}$} & & \multirow{4}{*}{ Synthetic dairy effluent } & BOD & $\begin{array}{l}\text { BOD = } \\
81.05 \%\end{array}$ & \multirow{4}{*}{$\begin{array}{l}\text { Sumithrabh } \\
\text { ai et al., } \\
\text { (2016) }\end{array}$} \\
\hline & & & COD & $\begin{array}{l}\text { COD = } \\
82.86 \%\end{array}$ & \\
\hline & & & $\mathrm{NO}_{3}^{-}$ & $\mathrm{NO}_{3}^{-}=77 \%$ & \\
\hline & & & $\mathrm{PO}_{4}{ }^{3-}$ & $\begin{array}{l}\mathrm{PO}_{4}^{3-}= \\
69.05 \%\end{array}$ & \\
\hline \multirow{3}{*}{$\begin{array}{l}\text { Scenedesmus } \\
\text { rubescens }\end{array}$} & \multirow{3}{*}{$\begin{array}{l}\text { Twin-layer } \\
\text { system }\end{array}$} & \multirow[b]{2}{*}{$\begin{array}{l}\text { Synthetic secondary } \\
\text { wastewater }\end{array}$} & $\mathrm{NO}_{3}^{-}$ & $\mathrm{NO}_{3}^{-}=95 \%$ & \multirow[b]{2}{*}{$\begin{array}{l}\text { Shi et al., } \\
\text { (2007) }\end{array}$} \\
\hline & & & $\begin{array}{l}\mathrm{NH}_{4}^{+} \\
\mathrm{PO}_{4}{ }^{3-}\end{array}$ & $\begin{array}{l}\mathrm{NH}_{4}{ }^{+}=96 \% \\
\mathrm{PO}_{4}{ }^{3-}=89 \%\end{array}$ & \\
\hline & & Municipal wastewater & $\mathrm{NO}_{3}^{-}$ & $\mathrm{NO}_{3}^{-}=96 \%$ & $\begin{array}{l}\text { Shi et al., } \\
(2007)\end{array}$ \\
\hline Scenedesmus & Chitosan & Synthetic 2 f medium with 44 & $\mathrm{NO}_{3}^{-}$ & $\mathrm{NO}_{3}^{-}=70 \%$ & Fierro et al., \\
\hline
\end{tabular}




\begin{tabular}{c|c|c|c|c|c}
\hline $\begin{array}{c}\text { Microalgae } \\
\text { Species }\end{array}$ & $\begin{array}{c}\text { Immobilizing } \\
\text { material }\end{array}$ & Source of the wastewater & Parameter & $\begin{array}{c}\text { Removal } \\
\text { efficiency } \\
(\%)\end{array}$ & Ref. \\
\hline \multirow{2}{*}{$\mathrm{sp}}$. & beads & $\begin{array}{c}\mathrm{mg} / \mathrm{L} \text { nitrate and } 6 \mathrm{mg} / \mathrm{L} \\
\text { phosphate }\end{array}$ & $\mathrm{PO}_{4}{ }^{3-}$ & $\mathrm{PO}_{4}{ }^{3-}=94 \%$ & $(2008)$ \\
\cline { 2 - 4 } & $\begin{array}{c}\text { Sodium } \\
\text { alginate } \\
\text { sheets }\end{array}$ & Domestic secondary effluent & $\mathrm{NH}_{4}{ }^{+}$ & $\mathrm{NH}_{4}{ }^{+}=100 \%$ & \multirow{2}{*}{$\begin{array}{c}\text { Zhang et al. } \\
(2008)\end{array}$} \\
\hline
\end{tabular}

\section{Factors affecting nutrients removal from wastewater in immobilized systems}

There are several factors that influence nutrients removal from wastewater in immobilized systems. These include the thickness of immobilized media, concentration of microalgae, and amount of beads.

\section{The thickness of immobilized media}

Zhang et al. (2008) investigated the effect of the thickness of Scenedesmus sp. immobilized gel on the uptake of nitrogen and phosphorus. They found out that gel thickness up to $3 \mathrm{~mm}$ is removed nutrients efficiently with higher algal biomass. Another studied by Hameed (2007) reported that small size beads with about $2.8 \mathrm{~mm}$ in diameter demonstrated higher removal efficiency for nitrate and phosphate than others beads ( 4 and $6 \mathrm{~mm}$ in diameter) in $48 \mathrm{~h}$. Low thickness media facilitate convective transport of nutrients between the gel media and the nutrient environment.

\section{The concentration of immobilized algae}

Too high cell density in gel results in low removal efficiency (Zhang et al., 2008). Sodium alginate sheets ( $3 \mathrm{~mm}$ thickness) which containing microalgae with $2 \times 10^{8}$ cells achieved higher nutrient removal compared to other sheets with $1.33 \times 10^{8}$ and $3 \mathrm{x}$ $10^{8}$ cell counts. Compared to low $\left(3.5 \times 10^{5}\right.$ cells $)$ and high $\left(3.10 \times 10^{6}\right.$ cells/bead $)$ cell stocking, calcium alginate beads containing microalgae cells $\left(1.5 \times 10^{6}\right.$ cells/bead $)$ demonstrated a greater capacity to effectively remove $\mathrm{NH}_{4}{ }^{+}$and $\mathrm{PO}_{4}{ }^{3-}$ from primary treated domestic wastewater (Hameed, 2007). They revealed that increasing cells stocking in beads causes leakage problems and affects the removal efficiency of target pollutants from wastewater. According to Jimenez-Perez et al. (2004), superconcentrated cells stockings may restrict to some extent the nutrient diffusion through the gel pores.

\section{The quantity of beads in wastewater}

The removal of target pollutants from wastewater is influenced by the quantity of beads containing algae cells used in the wastewater treatment process. A more effective removal of nitrate and phosphate from wastewater was achieved by 11 beads of algae than 16, 32, and 64 beads (Hameed, 2007). Tam and Wong (2000) accomplished a great removal of $\mathrm{NH}_{4}{ }^{+}$and $\mathrm{PO}_{4}{ }^{3-}$ from synthetic primary treated domestic wastewater within $24 \mathrm{~h}$ in bioreactors having an optimal algal bead concentration of 12 beads $/ \mathrm{mL}$ equivalent to $1: 3$ algal beads: wastewater $\mathrm{v} / \mathrm{v}$. $\mathrm{NH}_{4}{ }^{+}$removal was significantly lower with 15 beads $/ \mathrm{mL}$ algal concentration. They discussed that excessive increase in the quantity of beads results in high-density beads structure that hinders light penetration into cells and subsequently enhances self-shading effects, which limits the metabolic activities of microalgae. Moreover, the high concentration of beads results in the 
settling of beads at the bottom of the reactor due to ineffective air distribution to fluidize them.

\section{Current commercial-scale in wastewater treatment systems using suspended free- cells of microalgae}

There are several companies used algae for wastewater treatment practices worldwide for the commercial and industrial application. The companies that involve in algae-based energy (biogas) and fuel (biofuel and biodiesel) research are Algae Enterprises (Australia), Aquanos Energy Ltd. (Israel) and Fcc Aqualia (Spain). Adequate mass cultivation and harvesting of algae for algae biomass in wastewater is important to the aforementioned companies to achieve commercial viability. This is because the biomass can be used as feedstock for any biogas, biofuel, biodiesel or biofertilizer. According to the Website of these companies, they have developed different systems to treat wastewater using algae.

Algae Enterprises collaborated with the main shareholder, namely Sustainability Ventures Group have developed a technology, known as Photoluminescent Algae System (PAS) comprises of thin plastics embedded with fluorescent dyes in Australia (http://www.algaeenterprises.com/wastewater-treatment). Based on this system, the growth of algae is improved by fine-tuning the colors and wavelengths of incoming abundant sunlight that reaches the algae inside. This sustainable technology uses alga to remove pollutants including excessive nutrients from dairy wastewater in the presence of sunlight. In addition, this technology also integrates with the anaerobic digester to generate electricity through anaerobic digestion of harvested algae biomass to produce biogas with methane as the main component which can be used as a renewable source. Nutrient-rich residue materials (fertilizer product) resulting from the biogas generation are used for agriculture to produce even more algae. Meanwhile, the treated waste streams are recycled for irrigation and other purposes on the dairy farm.

Aquonos Energy Ltd developed a novel algae-based wastewater treatment system in Israel (https://finder.startupnationcentral.org/company_page/aquanos). The Aquanos system is characterized by lower energy consumption than conventional wastewater treatment system. Basically, this system consists of three distinct but interrelated processes namely, anaerobic treatment, aerobic treatment and separation of solid (algae) from treated effluent. The first stage is the anaerobic treatment of the incoming wastewater. The purpose of anaerobic treatment is to reduce the organic load to the downstream aerobic processes and at the same time to produce biogas for energy recovery as well as to produce $\mathrm{CO}_{2}$. The second stage is the aerobic treatment of anaerobic effluent which takes place in the fix film aerobic system. The aerobic system is aerated by a stream of oxygen-rich algae which are grown in the separate raceway pond. Based on this system, the algae are grown in the raceway pond to produce high dissolved oxygen in the liquid. Then, this liquid recirculates through the fix film system supplying oxygen for bacterial decomposition of organic pollutants. At the end of the process, namely solid separation stage is where the excessive algae and excessive biomass are separated from treated effluent. The treated effluent is discharged into the environment and reused for agriculture, while the excessive biomass is returned to the anaerobic stage to produce additional biogas. The end product is high-quality effluent produce using less energy than the conventional system as well as resource harvesting through the production of high quality and high-level algae by-product. 
Fcc Aqualia in Spain partnered with University of Southampton (England), BDI (Austria), Frounhofer Society (German), HyGear (Netherlands) and Volkswagen (German) companies under All-Gas project for developing a new pond system to cultivate algae using nutrients present in the wastewater at wastewater treatment plant to produce biodiesel and biogas (methane) made from biomass to power vehicles (https://www.power-technology.com/uncategorised/newsaqualias-biofuel-projectproduces-first-algae-biomass-in-spain/). Basically, they have two different processes of the sequential order under this project, namely All-Gas Alternative 1 (post lipid extraction) and All-Gas Alternative 2 (pre lipid extraction) to obtain biodiesel, biomethane, and biogas. For All-Gas Alternative 1, the incoming raw wastewater is pretreated and the effluent is discharged into raceway pond containing algae culture. After that, the dense algae culture is harvested and the treated wastewater is discharged to the environment, while the harvested algae biomass undergoes the anaerobic process in anaerobic digester. The biogas is produced during anaerobic treatment and the further undergo pre-treatment and upgrading process to obtain pure methane and $\mathrm{CO}_{2}$, respectively. The biomethane are stored in the refueling station, while the $\mathrm{CO}_{2}$ is supplied to the algae culture grown in the aforementioned raceway pond to promote high growth rate and yield of algae. In addition, the remaining residue after anaerobic treatment in anaerobic digester undergoes dewatering method to concentrate prior to the extraction process to produce biodiesel and biofertilizer (remain residue). The All-Gas Alternative 2 has the same process of sequential order as All-Gas Alternative 1 but the lipid extraction process occurs directly upon harvesting. They produce approximately $200000 \mathrm{~L}$ of biodiesel and $600000 \mathrm{~m}^{3}$ of biomethane per year from approximately 3000 $\mathrm{kg}$ of dry algae with 20 percent of oil content after grown in ponds of 10 hectares. They claim that the Volkswagen vehicles that have been power using algae-based biogas emit zero emissions. The European Commission contributed about 12 million euro ( $\$ 15.9$ million USD) for this project with the target of at least $10 \%$ renewable energy used in their transport sectors by 2020. So far, there is none company use immobilized algae to treat wastewaters commercially.

\section{Wastewater treatment challenges using suspended free-cells of microalgae}

To date, limited investments have been pushed into the development of commercialscale algal wastewater treatment plant, and this has been the result of technical challenges relating to scale-up feasibility, harvesting, and dewatering of biomass. Practical application of current emerging technologies is still in its infancy, with most of the technologies validated only at the laboratory scale.

Most algae are cultivated in closed PBRs for phycoremediation and biomass production (Kamarudin et al., 2015; Lage et al., 2018). The expensive culture system with high capital cost and energy requirements for mixing and gas exchange together with the cost of harvesting to achieve feasible algal solid concentration has constrained the integration of microalgal system with wastewater treatment at large-scale levels. This can partially be addressed through the use of other systems such as open raceway pond. However, environmental factors such as temperature fluctuation, weather influence, and light penetration can affect the efficiency of phycoremediation and productivity of biomass.

Algae require sufficient amount of $\mathrm{CO}_{2}$ for growth. Thus, low-cost approach using flue gas from power plants as carbon source can be applied. However, the high 
concentration of substances like nitrogen oxide, sulfur oxide, and heavy metals presents in the flue gas causes the medium very acidic for algae cultivation. As a result, algae cultivation is prone to contamination and inhibition, resulting in low wastewater treatment efficiency and low biomass production. Therefore, the flue gas can be pretreated to minimize contamination before exposure to the microalgae cells. The pretreatment process could significantly increase the total operation cost.

Kamarudin et al. (2015) propose the following for consideration in algal POME treatment: (i) pre-treatment of wastewater to remove growth inhibitors; (ii) feasible and economical method for algae cultivation and biomass harvesting; and (iii) selection of suitable microalgae strains.

High concentration of nutrients such as ammonium can inhibit the growth of algae and lead to poor wastewater treatment efficiency. According to Cai et al. (2013), nitrogen in the form of ammonium is the most preferred source for effective and rapid growth of algae due to effective redox reaction during nitrogen assimilation and the less energy requirement for the reaction to occur.

The selection of suitable algae for effective phycoremediation and $\mathrm{CO}_{2}$ fixation is critical to the process development as microalgae cells have different tolerance to the range of pollutants, $\mathrm{CO}_{2}$ concentration, and also the culture condition. According to Choul-gyun (2002), algal strain such Chlorella kessleri is capable of removing various concentration of nitrogen up to $1400 \mathrm{mg} / \mathrm{L}$ indicating that this algal strain has a high tolerance to nitrogen.

Numerous studies have been conducted by researchers around the world on the application of algae for phycoremediation and biomass production for sustainable bioproducts production. With further research and development targeted at addressing some of the above-mentioned challenges, commercial-scale wastewater treatment using algae can be achieved.

\section{Wastewater treatment challenges using immobilized microalgae}

There are some challenges with the use of polymer immobilized microalgae systems according to Cai et al. (2013). These are (i) the capability of the system to remove pollutants present in wastewater effectively; (ii) cost of polymer and subsequent immobilization process; (iii) chemical forces and interactions between the immobilization matrix and the cell wall may result in abiotic stresses (iv) limited diffusion of substrate or fluid like wastewater, metabolic products, oxygen and $\mathrm{CO}_{2}$ to and from the cells in the polymeric matrix; (v) sufficient light penetration into the polymer matrix containing algal cells; and (vi) the metabolism of microalgae is affected by its confinement in a limited space. However, these issues can be addressed by combining optimized immobilization matrices with smart bioreactor designs.

\section{Conclusion}

Effective wastewater treatment is vital in order to improve the quality of wastewaters effluent which must be met the regulations standards set by local authorities before discharge. Thus, the use of suspended free-cells microalgae culture emerges as a viable option in future and can be explored to treat wastewaters containing nutrients sources with simultaneous $\mathrm{CO}_{2}$ capture which are required for growth and support microalgae cultivation. For that reason, the use of biochemical abilities of suspended free-cells 
microalgae culture is a popular approach to be used as a tertiary treatment in conventional wastewater treatment which can remove nutrient and BOD efficiently in the engineered system like high rate algal ponds. Most recent studies have highlighted the various advantages of immobilized microalgae in carriers as compared to suspended free-cells microalgae culture for removal of nutrients, BOD and COD from wastewaters. For instance, application of immobilized algae to treat wastewaters capable to reduce the cost of all process by circumventing the need for downstream processes using dewatering and harvesting methods to separate the biomass and treated effluent. In contrast, a very costly dewatering and harvesting methods are required to obtain biomass derived from free-cells microalgae culture due to their dilute nature and small in size. Therefore, the immobilized microalgae in capsules not only ease the harvesting process but also increase the efficiency of wastewaters treatment with $\mathrm{CO}_{2}$ sequestration and bioproduct generation derived from microalgae biomass. The removal of nutrient, BOD and COD from wastewaters using immobilized microalgae cells has been one of the major interesting research subjects carried out by researchers globally due to its environment-friendly approach for sustainable development in the future.

Acknowledgements. The authors thank the support of any parties involved in this project especially Universiti Putra Malaysia for providing the equipment and research facilities to conduct this project. The financial contribution provided by MyBrain15 Scheme is acknowledged.

\section{REFERENCES}

[1] Abdel-Raouf, N., Al-Homaidan, A. A., Ibraheem, I. B. M. (2012): Microalgae and wastewater treatment. - Saudi Journal of Biological Sciences 19(3): 257-275.

[2] Abou-Shanab, R. A. I., Ji, M. K., Kim, H. C., Paeng, K. J., Jeon, K. J. (2013): Microalgal species growing on piggery wastewater as a valuable candidate for nutrient removal and biodiesel production. - Journal of Environmental Management 115: 257-264.

[3] Ahmad, F., Khan, A. U., Yasar, A. (2013): Comparative Phycoremediation of Sewage Water by Various Species of Algae. - Proceedings of the Pakistan Academy of Sciences 50(2): 131-139.

[4] Ahmed, S. G. K. A. (2014): Dairy Wastewater Treatment Using Microalgae in Karbala City, Iraq. - International Journal of Environment, Ecology, Family and Urban Studies (IJEEFUS) 4(2): 13-22.

[5] Amaro, H. M., Guedes, A. C., Malcata, F. X. (2011): Advances and Perspectives in Using Microalgae to Produce Biodiesel. - Applied Energy 88(10): 3402-3410.

[6] Aravantinou, A. F., Theodorakopoulos, M. A., Manariotis, I. D. (2013): Selection of microalgae for wastewater treatment and potential lipids production. - Bioresource Technology 147: 130-134.

[7] Azarpira, H., Behdarvand, P., Dhumal, K., Pondhe, G. (2014): Potential use of cyanobacteria species in phycoremediation of municipal wastewater. - International Journal of Bioscience 4(4): 105-111.

[8] Aziz, M. A., Ng, W. J. (1992): Feasibility of wastewater treatment using the activatedalgae process. - Bioresource Technology 40(3): 205-208.

[9] Barakat, M. A. (2011): New Trends in Removing Heavy Metals from Industrial Wastewater. - Arabian Journal of Chemistry 4(4): 361-377.

[10] Bhatt, N. C., Panwar, A., Bisht, T. S., Tamta, S. (2014): Coupling of algal biofuel production with wastewater. - Scientific World Journal : 1-10.

[11] Boopathy, R. (2000): Factors limiting bioremediation technologies. - Bioresource Technology 74(1): 63-67. 
[12] Brennan, L., Owende, P. (2010): Biofuels from Microalgae-A Review of Technologies for Production, Processing, and Extractions of Biofuels and Co-Products. - Renewable and Sustainable Energy Reviews 14(2): 557-577.

[13] Cai, T., Park, S. Y., Li, Y. (2013): Nutrient Recovery from Wastewater Streams by Microalgae: Status and Prospects. - Renewable and Sustainable Energy Reviews 19: 360369.

[14] Can, S. S., Demir, V., Korkmaz, S. A., Can, E. (2013): Treatment of domestic waste water with Botryococcus braunii (Cholorophyceae). - Journal Of Food Agriculture And Environment 11(October): 3-5.

[15] Chan, Y. J., Chong, M. F., Law, C. L., Hassell, D. G. (2009): A Review on AnaerobicAerobic Treatment of Industrial and Municipal Wastewater. - Chemical Engineering Journal 155(1-2): 1-18.

[16] Chinnasamy, S., Bhatnagar, A., Hunt, R. W., Das, K. C. (2010): Microalgae cultivation in a wastewater dominated by carpet mill effluents for biofuel applications. - Bioresour. Technol. 101: 3097-3105.

[17] Chojnacka, K., Noworyta, A. (2004): Evaluation of Spirulina sp. growth in photoautotrophic, heterotrophic and mixotrophic cultures. - Enzyme and Microbial Technology 34(5): 461-465.

[18] Choul-gyun, L., Lee, K. (2002): Nitrogen Removal from Wastewaters by Microalgae Without Consuming Organic Carbon Sources. - J. Microbiol. Biotechnol 12: 979-985.

[19] Clarens, A. F., Ressureccion, E. P., White, M. A., Colosi, L. M. (2009): Environmental life cycle comparison of algae to other bioenergy feedstocks. - Environmental Science and Technology 44(5): 1813-1819.

[20] Cohen, Y. (2001): Biofiltration - The treatment of fluids by microorganisms immobilized into the filter bedding material: a review. - Bioresource Technology 77(3): 257-274.

[21] Costa, J. A. V., de Morais, M. G. (2013): An Open Pond System for Microalgal Cultivation. - In: Pandey, A., Lee, D. J., Chisti, Y., Soccol, C. Y. (eds.) Biofuels from Algae, Elsevier B.V., San diego.

[22] De-Bashan, L. E., Hernandez, J. P., Morey, T., Bashan, Y. (2004): Microalgae growthpromoting bacteria as "helpers" for microalgae: A novel approach for removing ammonium and phosphorus from municipal wastewater. - Water Research 38(2): 466474.

[23] De-Bashan, L. E., Bashan, Y. (2010): Immobilized microalgae for removing pollutants: Review of practical aspects. - Bioresource Technology 101(6): 1611-1627.

[24] Delgadillo-Mirquez, L., Lopes, F., Taidi, B., Pareau, D. (2016): Nitrogen and phosphate removal from wastewater with a mixed microalgae and bacteria culture. - Biotechnology Reports 11: 18-26.

[25] Ding, G. T, Yaakob, Z., Takriff, M. S., Salihon, J., Abd Rahaman, M. S. (2016): Biomass Production and Nutrients Removal by a Newly-Isolated Microalgal Strain Chlamydomonas sp. in Palm Oil Mill Effluent (POME). - International Journal of Hydrogen Energy 41(8): 4888-4895.

[26] Dominic, V., Murali, S., Nisha, M. (2009): Phycoremediation efficiency of three microalgae Chlorella vulgaris, Synechocytis salina and Gloeocapsa gelatinosa. - SB Academic Review 16(1-2): 138-146.

[27] Driscoll, C. T., Whitall, D., Aber, J., Boyer, E., Castro, M., Cronan, C., Goodale, C., Groffman, P., Hopkinson, C., Lambert, K., Lawrence, G., Ollinger, S. (2003): Nitrogen Pollution in the Northeastern United States: Sources, Effects, and Management Options. BioScience 53(4): 357-374.

[28] Eroglu, E., Agarwal, V., Bradshaw, M., Chen, X., Smith, S. M., Raston, C. L., Swaminathan Iyer, K. (2012): Nitrate Removal from Liquid Effluents Using Microalgae Immobilized on Chitosan Nanofiber Mats. - Green Chemistry 14(10): 2682.

[29] Eroglu, E., Smith, S. M., Raston, C. L. (2015): Application of Various Immobilization Techniques for Algal Bioprocesses. - In: Moheimani, N. R., McHenry, M. P., de Boer, 
K., Bahri, P. (eds.) Biomass and Biofuels from Microalgae, Springer International Publishing, Murdoch.

[30] Fierro, S., del Pilar Sánchez-Saavedra, M., Copalcúa, C. (2008): Nitrate and phosphate removal by chitosan immobilized Scenedesmus. - Bioresource Technology 99(5): 12741279.

[31] Ganeshkumar, V., Subashchandrabose, S. R., Dharmarajan, R., Venkateswarlu, K., Naidu, R., Megharaj, M. (2018): Use of mixed wastewaters from piggery and winery for nutrient removal and lipid production by Chlorella sp. MM3. - Bioresource Technology 256(February): 254-258.

[32] Gani, P., Sunar, N. M., Latiff, A. A., Kamaludin, N. S., Parjo, U. K., Emparan, Q. (2015a): Experimental Study for Phycoremediation of Botryococcus sp. on Greywater. Applied Mechanics and Materials 773-774: 1312-1317.

[33] Gani, P., Sunar, N. M., Latiff, A. A., Joo, I. T. K., Parjo, U. K., Emparan, Q., Er, C. M. (2015b): Phycoremediation of Dairy Wastewater by Using Green Microlgae: Botryococcus sp. - Applied Mechanics and Materials 773-774: 1318-1323.

[34] Gani, P., Sunar, N. M., Matias-peralta, H., Latiff, A. A. A., Kalthsom, U. K., Razak, A. R. A. (2015c): Phycoremediation of Wastewaters and Potential Hydrocarbon from Microalgae: A Review. - Advances in Environmental Biology 9(20):1-8.

[35] Gokulan, R., Sathish, N., Praveen Kumar, R. (2013): Treatment of grey water using hydrocarbon producing Botryococcus braunii. - International Journal of ChemTech Research 5(3): 1390-1392.

[36] Hadiyanto, H., Christwardana, M., Soetrisnanto, D. (2013): Phytoremediations of Palm Mill Effluent (POME) by Using Aquatic Plants and Mircoalgae for Biomass Production. - Journal of Environmental Science and Technology 6(2): 79-90.

[37] Hadiyanto, H., Soetrisnanto, D., Christwardhana, M. (2014): Phytoremediation of Palm Oil Mill Effluent Using Pistia Stratiotes Plant and Algae. - International Journal of Engineering (IJE) 27(12): 1809-1814.

[38] Hameed, M. S. A. (2007): Effect of algal density in bead, bead size and bead concentrations on wastewater nutrient removal. - African Journal of Biotechnology 6(May): 1185-1191.

[39] Hernandez, J. P., De-Bashan, L. E., Bashan, Y. (2006): Starvation enhances phosphorus removal from wastewater by the microalga Chlorella spp. co-immobilized with Azospirillum brasilense. - Enzyme and Microbial Technology 38(1-2): 190-198.

[40] https://finder.startupnationcentral.org/company_page/aquanos.

[41] http://www.algaeenterprises.com/wastewater-treatment.

[42] https://www.power-technology.com/uncategorised/newsaqualias-biofuel-projectproduces-first-algae-biomass-in-spain.

[43] Huang, G., Wang, Y. (2003): Nitrate and Phosphate Removal by Co-immobilized Chlorella pyrenoidosa and Activated Sludge at Different pH Values. - Water Qual. Res. J. Canada 38(3): 541-551.

[44] Huang, G., Chen, F., Wei, D., Zhang, X., Chen, G. (2010): Biodiesel production by microalgal biotechnology. - Applied Energy 87(1): 38-46.

[45] Hultberg, M., Carlsson, A. S., Gustafsson, S. (2013): Treatment of drainage solution from hydroponic greenhouse production with microalgae. - Bioresource Technology 136: 401406.

[46] Huy, M., Kumar, G., Kim, H. W., Kim, S. H. (2018): Photoautotrophic cultivation of mixed microalgae consortia using various organic waste streams towards remediation and resource recovery. - Bioresource Technology 247(June): 576-581.

[47] Jaysudha, S., Sampathkumar, P. (2014): Nutrient removal from tannery by free and immobilized cells of marine microalgae Chlorella salina. - International Journal of Environmental Biology 4(1): 21-26.

[48] Ji, M., Abou-Shanab, R. A. I., Hwang, J., Timmes, T. C., Kim, H., Oh, Y., Jeon, B. (2013): Removal of Nitrogen and Phosphorus from Piggery Wastewater Effluent Using 
the Green Microalga Scenedesmus obliquus. - Journal of Environmental Engineering 139(9): 1198-1205.

[49] Ji, X., Jiang, M., Zhang, J., Jiang, X., Zheng, Z. (2018): The interactions of algae-bacteria symbiotic system and its effects on nutrients removal from synthetic wastewater. Bioresource Technology 247(July): 44-50.

[50] Jimenez-Perez, M. V., Sanchez-Castillo, P., Romera, O., Fernandez-Moreno, D., PerezMartinez, C. (2004): Growth and nutrient removal in free and immobilized planktonic green algae isolated from pig manure. - Enzyme and Microbial Technology 34(5): 392398.

[51] Kamarudin, K. F., Tao, D. G., Yaakob, Z., Takriff, M. S., Rahaman, M. S. A., Salihon, J. (2015): A Review on Wastewater Treatment and Microalgal By-Product Production with a Prospect of Palm Oil Mill Effluent (POME) Utilization for Algae. - Der Pharma Chemica 7(7): 73-89.

[52] Kamyab, H., Din, M. F. M., Lee, C. T., Ponraj, M., Soltani, M., Mohamad, S. E., Roudi, A. M. (2014): Micro-macro Algal Mixture as a Promising Agent for Treating POME Discharge and Its Potential Use as Animal Feed Stock Enhancer. - Journal Teknologi (Sciences and Engineering) 68(5): 1-4.

[53] Kamyab, H., Din, M. F. M., Keyvanfar, A., Majid, M. Z. A., Talaiekhozani, A., Shafaghat, A., Lee, C. T., Shiun, L. J., Ismail, H. H. (2015): Efficiency of Microalgae Chlamydomonas on the Removal of Pollutants from Palm Oil Mill Effluent (POME). Energy Procedia 75: 2400-2408.

[54] Kamyab, H., Chelliapan, S., Din, M. F. M., Shahbazian-Yassar, R., Rezania, S., Khademi, T., Kumar, A., Azimi, M. (2017): Evaluation of Lemna Minor and Chlamydomonas to Treat Palm Oil Mill Effluent and Fertilizer Production. - Journal of Water Process Engineering 17(May): 229-236.

[55] Katarzyna, L., Sai, G., Avijeet Singh, O. (2015): Non-enclosure methods for nonsuspended microalgae cultivation: Literature review and research needs. - Renewable and Sustainable Energy Reviews 42: 1418-1427. https://doi.org/10.1016/j.rser.2014.11.029.

[56] Komolafe, O., Velasquez Orta, S. B., Monje-Ramirez, I., Noguez, I. Y. Y., Harvey, A. P., Ledesma, M. T. O. (2014): Biodiesel Production from Indigenous Microalgae Grown in Wastewater. - Bioresource Technology 154(November): 297-304.

[57] Kothari, R., Prasad, R., Kumar, V., Singh, D. P. (2013): Production of biodiesel from microalgae Chlamydomonas polypyrenoideum grown on dairy industry wastewater. Bioresource Technology 144: 499-503.

[58] Kotteswari, M., Murugesan, S., Rk, R. (2012): Phycoremediation of Dairy Effluent by using the Microalgae Nostoc sp. - International Journal of Environmental Research and Development 2(1): 35-43.

[59] Kshirsagar, D. A. (2013): Bioremediation of Wastewater By Using Microalgae: an Experimental Study. - International Journal of Life Science Biotechnology and Pharma Research 2(3): 339-346.

[60] Kumar, M., Sharma, M. P., Dwivedi, G. (2013): Algae Oil as Future Energy Source in Indian Perspective. - International Journal of Renewable Energy Research 3(4): 913-921.

[61] Kumar, P. K., Krishna, S. V., Verma, K., Pooja, K., Bhagawan, D., Himabindu, V. (2018): Phycoremediation of sewage wastewater and industrial flue gases for biomass generation from microalgae. - South African Journal of Chemical Engineering 25: 133146.

[62] Lage, S., Gojkovic, Z., Funk, C., Gentili, F. (2018): Algal Biomass from Wastewater and Flue Gases as a Source of Bioenergy. - Energies 11(3): 664.

[63] Lam, M. K., Lee, K. T. (2011): Renewable and sustainable bioenergies production from palm oil mill effluent (POME): Win-win strategies toward better environmental protection. - Biotechnology Advances 29(1): 124-141. 
[64] Lau, P. S., Tam, N. F. Y., Wong, Y. S. (1997): Wastewater Nutrients (N and P) Removal by Carrageenan and Alginate Immobilized Chlorella vulgaris. - Environmental Technology 18(9): 945-951.

[65] Lim, S. L., Chu, W. L., Phang, S. M. (2010): Use of Chlorella vulgaris for bioremediation of textile wastewater. - Bioresource Technology 101(19): 7314-7322.

[66] Loh, S. K., Lai, M. E., Ngatiman, M. (2013): Zero Discharge Treatment Technology of Palm Oil Mill Effluent. - Journal of Oil Palm Research 25(3): 273-281.

[67] Malik, N. (2002): Biotechnological potential of immobilised algae for wastewater N, P and metal removal: a review. - BioMetals 15: 377-390.

[68] Marella, T. K., Parine, N. R., Tiwari, A. (2018): Potential of diatom consortium developed by nutrient enrichment for biodiesel production and simultaneous nutrient removal from waste water. - Saudi Journal of Biological Sciences 25(4): 704-709.

[69] Martínez, M. E., Jiménez, J. M., El Yousfi, F. (1999): Influence of phosphorus concentration and temperature on growth and phosphorus uptake by the microalga Scenedesmus obliquus. - Bioresource Technology 67(3): 233-240.

[70] Mata, T. M., Martins, A. A., Caetano, N. S. (2010): Microalgae for Biodiesel Production and Other Applications: A Review. - Renewable and Sustainable Energy Reviews 14(1): 217-232.

[71] Mata, T. M., Melo, A. C. Simões, M., Caetano, N. S. (2012): Parametric study of a brewery effluent treatment by microalgae Scenedesmus obliquus. - Bioresource Technology 107(January): 151-158.

[72] McElwee, K., Baker, J., Clair, D. (2006): Pond Fertilization: Ecological Approach and Practical Application. - John Wiley \& Sons.

[73] Michels, M. H. A., Vaskoska, M., Vermuë, M. H., Wijffels, R. H. (2014): Growth of Tetraselmis Suecica in a Tubular Photobioreactor on Wastewater from a Fish Farm. Water Research 65: 290-296.

[74] Mohan, N., Balasubramanian, N., Subramanian, V. (2001): Electrochemical treatment of simulated textile effluent. - Chemical Engineering and Technology 24(7): 749-753.

[75] Moreno-Garrido, I. (2008): Microalgae immobilization: Current techniques and uses. Bioresource Technology 99(10): 3949-3964.

[76] Murugesan, S., Dhamotharan, R. (2009): Bioremediation of thermal wastewater by Pithophora sp. - Current World Environment 4(1): 137-142.

[77] Olguin, E. J. (2003): Phycoremediation: Key issues for cost-effective nutrient removal processes. - Biotechnology Advances 1(2): 81-91.

[78] Oswald, W. J., Gotaas, H. B., Golueke, C. G., Kellen, W. R., Gloyna, E. F., Hermann, E. R. (1957): Algae in Waste Treatment. - Sewage and Industrial Wastes 29(54): 437-457.

[79] Packer, M. (2009): Algal capture of carbon dioxide; biomass generation as a tool for greenhouse gas mitigation with reference to New Zealand energy strategy and policy. Energy Policy 37(9): 3428-3437.

[80] Paskuliakova, A., McGowan, T., Tonry, S., Touzet, N. (2018a): Microalgal bioremediation of nitrogenous compounds in landfill leachate - The importance of micronutrient balance in the treatment of leachates of variable composition. - Algal Research 32: 162-171.

[81] Paskuliakova, A., McGowan, T., Tonry, S., Touzet, N. (2018b): Phycoremediation of landfill leachate with the chlorophyte Chlamydomonas sp. SW15aRL and evaluation of toxicity pre and post treatment. - Ecotoxicology and Environmental Safety 147: 622-630.

[82] Phang, S. M., Ong, K. C. (1988): Algal Biomass Production in Digested Palm Oil Mill Effluent. - Biological Wastes 25: 77-191.

[83] Pittman, J. K., Dean, A. P., Osundeko, O. (2011): The potential of sustainable algal biofuel production using wastewater resources. - Bioresource Technology 102(1): 17-25.

[84] Poo, K., Son, E., Chang, J., Ren, X., Choi, Y., Chae, K. (2018): Biochars derived from wasted marine macro-algae (Saccharina japonica and Sargassum fusiforme) and their 
potential for heavy metal removal in aqueous solution. - J. Environ. Manage. 206: 364372.

[85] Raj, A. S. (2015): Botryococcus braunii as a Phycoremediation Tool for the Domestic Waste Water Recycling from Cooum River, Chennai, India. - Journal of Bioremediation \& Biodegradation 6(3): 1-9.

[86] Rajkumar, R., Takriff, M. S. (2015): Nutrient Removal from Anaerobically Treated Palm Oil Mill Effluent by Spirulina Platensis and Scenedesmus Dimorphus. - Der Pharmacia Lettre 7(7): 416-421.

[87] Rao, P., Kumar, R. R., Raghavan, B., Sivasubramanian, V. (2011): Application of phycoremediation technology in the treatment of wastewater from a leather-processing chemical manufacturing facility. - Water SA 37(1): 7-14.

[88] Rawat, I., Kumar, R. R., Mutanda, T., Bux, F. (2011): Dual Role of Microalgae: Phycoremediation of Domestic Wastewater and Biomass Production for Sustainable Biofuels Production. - Applied Energy 88(10): 3411-3424.

[89] Riano, B., Molinuevo, B., Garcia-Gonzalez, M. C. (2011): Treatment of fish processing wastewater with microalgae-containing microbiota. - Bioresource Technology 102(23): 10829-10833.

[90] Rugnini, L., Costa, G., Congestri, R., Antonaroli, S., Sanità di Toppi, L., Bruno, L. (2018): Phosphorus and metal removal combined with lipid production by the green microalga Desmodesmus sp.: An integrated approach. - Plant Physiology and Biochemistry 125: 45-51.

[91] Sacristán de Alva, M., Luna-Pabello, V. M., Cadena, E., Ortíz, E. (2013): Green Microalga Scenedesmus Acutus Grown on Municipal Wastewater to Couple Nutrient Removal with Lipid Accumulation for Biodiesel Production. - Bioresource Technology 146: 744-748.

[92] Safonova, B. E., Kvitko, K. V., Iankevitch, M. I., Surgko, L. F., Afti, I. A., Reisser, W., (2004): Biotreatment of Industrial Wastewater by Selected Algal-Bacterial Consortia. Microb. Enhanc. Oil Recover.: 347-353.

[93] Sahu, O. (2014): Reduction of Heavy Metals from Waste Water by Wetland. International Letters of Natural Sciences 7: 35-43.

[94] Schoeman, J. J., Steyn, A. (2003): Nitrate removal with reverse osmosis in a rural area in South Africa. - Desalination 155: 15-26.

[95] Selvam, T. B. T., Renganathan, R., Takriff, M. S. (2015): Nutrient Removal of POME Using POME Isolated Microalgae Strain. - Advanced Materials Research 1113: 364-369.

[96] Sengar, R. M., Singh, K. K., Singh, S. (2011): Application of phycoremediation technology in the treatment of sewage water to reduce pollution load. - Indian journal Science Resource 2(4): 33-39.

[97] Sharma, G., Khan, S. (2013): Bioremediation of Sewage Wastewater Using Selective Algae for Manure Production. - International Journal of Environmental Engineering and Management 4(6): 573-580.

[98] Sharma, Y. C., Singh, B., Korstad, J. (2011): A critical review on recent methods used for economically viable and eco-friendly development of microalgae as a potential feedstock for synthesis of biodiesel. - Green Chemistry 13(11): 2993-3006.

[99] Shi, J., Podola, B., Melkonian, M. (2007): Removal of nitrogen and phosphorus from wastewater using microalgae immobilized on twin layers: An experimental study. Journal of Applied Phycology 19(5): 417-423.

[100] Sirakov, I. N., Velichkova, K. N. (2014): Bioremediation of wastewater originate from aquaculture and biomass production from microalgae species - Nannochloropsis oculata and Tetraselmis chuii. - Bulgarian Journal of Agricultural Science 20(1): 66-72.

[101] Sivakumar, R., Rajendran, S. (2013): Role of Algae in Commercial Environment. International Research Journal of Environment Sciences 2(12): 81-83.

[102] Smith, V. (2003): Eutrophication of freshwater and coastal marine ecosystems a global problem. - Environmental Science and Pollution Research 10(2): 126-139. 
[103] Sperling, M. V., Chernicharo, C. A. D. L. (2005): Biological Wastewater Treatment in Warm Climate Regions. - IWA Publishing.

[104] Su, Y., Mennerich, A., Urban, B. (2011): Municipal wastewater treatment and biomass accumulation with a wastewater-born and settleable algal-bacterial culture. - Water Research 45(11): 3351-3358.

[105] Su, Y., Mennerich, A., Urban, B. (2012): Comparison of nutrient removal capacity and biomass settleability of four high-potential microalgal species. - Bioresource Technology 124: $157-162$.

[106] Sukačová, K., Trtílek, M., Rataj, T. (2015): Phosphorus removal using a microalgal biofilm in a new biofilm photobioreactor for tertiary wastewater treatment. - Water Research 71: 55-63.

[107] Sumithrabhai, K., Thirumarimurugan, M., Sivakumar, V. M., Sujatha, S. (2016): Expedient Study on Treatment of Dairy Effulent in Fluidized Bed Reactor Using Immobilized Microalgae. - International Journal of Advanced Engineering Technology 7(2): 231-235.

[108] Sydney, E. B., da Silva, T. E., Tokarski, A., Novak, A. C., de Carvalho, J. C., Woiciecohwski, A. L., Larroche, C., Soccol, C. R. (2011): Screening of microalgae with potential for biodiesel production and nutrient removal from treated domestic sewage. Applied Energy 88(10): 3291-3294.

[109] Tam, N. F. Y., Wong, Y. S. (2000): Effect of immobilized microalgal bead concentrations on wastewater nutrient removal. - Environmental Pollution 107(1): 145151.

[110] Udom, I., Zaribaf, B. H., Halfhide, T., Gillie, B., Dalrymple, O., Zhang, Q., Ergas, S. J. (2013): Harvesting microalgae grown on wastewater. - Bioresource Technology 139: 101-106.

[111] Vasilieva, S. G., Lobakova, E. S., Lukyanov, A. A., Solovchenko, A. E. (2016): Immobilized Microalgae in Biotechnology. - Moscow University Biological Sciences Bulletin 71(3): 170-176.

[112] Wang, L., Min, M., Li, Y., Chen, P., Chen, Y., Liu, Y., Wang, Y., Ruan, R. (2010): Cultivation of green algae Chlorella $s p$. in different wastewaters from municipal wastewater treatment plant. - Applied Biochemistry and Biotechnology 162(4): 11741186.

[113] Whangchenchom, W., Chiemchaisri, W., Tapaneeyaworawong, P., Powtongsook, S. (2014): Wastewater from instant noodle factory as the whole nutrients source for the microalga Scenedesmus sp. Cultivation. - Environmental Engineering Research 19(3): 283-287.

[114] Yadavalli, R., Heggers, G. R. V. N., Rao, G., Naga, V. (2013): Two Stage Treatment of Dairy Effluent Using Immobilized Chlorella Pyrenoidosa. - Journal of environmental health science \& engineering 11(1): 36.

[115] Zeng, X., Danquah, M. K., Zheng, C. R., Chen, X. D., Lu, Y. (2012): NaCS-PDMDAAC immobilized autotrophic cultivation of Chlorella $s p$. for wastewater nitrogen and phosphate removal. - Chemical Engineering Journal 187: 185-192.

[116] Zhang, E., Wang, B., Wang, Q., Zhang, S., Zhao, B. (2008): Ammonia-nitrogen and orthophosphate removal by immobilized Scenedesmus sp. isolated from municipal wastewater for potential use in tertiary treatment. - Bioresource Technology 99(9): 37873793.

[117] Zhou, W., Li, Y., Min, M., Hu, B., Zhang, H., Ma, X., Li, L., Cheng, Y., Chen, P., Ruan, R. (2012): Growing Wastewater-born Microalga Auxeno Chlorella Protothecoides UMN280 on Concentrated Municipal Wastewater for Simultaneous Nutrient Removal and Energy Feedstock Production. - Applied Energy 98: 433-440.

[118] Zhu, L., Wang, Z., Shu, Q., Takala, J., Hiltunen, E., Feng, P., Yuan, Z. (2013): Nutrient removal and biodiesel production by integration of freshwater algae cultivation with piggery wastewater treatment. - Water Research 47(13): 4294-4302. 